\title{
Interleukin-1 Receptor Accessory Protein Organizes Neuronal Synaptogenesis as a Cell Adhesion Molecule
}

\author{
Tomoyuki Yoshida, ${ }^{1,3}$ Tomoko Shiroshima, ${ }^{1}$ Sung-Jin Lee, ${ }^{1}$ Misato Yasumura, ${ }^{1}$ Takeshi Uemura, ${ }^{1}$ Xigui Chen, ${ }^{1}$ \\ Yoichiro Iwakura, ${ }^{2}$ and Masayoshi Mishina ${ }^{1}$ \\ ${ }^{1}$ Department of Molecular Neurobiology and Pharmacology, Graduate School of Medicine, University of Tokyo, Tokyo 113-0033, Japan, ${ }^{2}$ Center for \\ Experimental Medicine and Systems Biology, Institute of Medical Science, University of Tokyo, Tokyo 108-8639, Japan, and ${ }^{3}$ PRESTO (Precursory Research \\ for Embryonic Science and Technology), Japan Science and Technology Agency, Saitama 332-0012, Japan
}

Interleukin-1 receptor accessory protein (IL-1RAcP) is the essential component of receptor complexes mediating immune responses to interleukin-1 family cytokines. IL-1RAcP in the brain exists in two isoforms, IL-1RAcP and IL-1RAcPb, differing only in the C-terminal region. Here, we found robust synaptogenic activities of IL-1RAcP in cultured cortical neurons. Knockdown of IL-1RAcP isoforms in cultured cortical neurons suppressed synapse formation as indicated by decreases of active zone protein Bassoon puncta and dendritic protrusions. IL-1RAcP recovered the accumulation of presynaptic Bassoon puncta, while IL-1RAcPb rescued both Bassoon puncta and dendritic protrusions. Consistently, the expression of IL-1RAcP in cortical neurons enhances the accumulation of Bassoon puncta and that of IL-1RAcPb stimulated both Bassoon puncta accumulation and spinogenesis. IL-1RAcP interacted with protein tyrosine phosphatase (PTP) $\delta$ through the extracellular domain. Mini-exon peptides in the Ig-like domains of PTP $\delta$ splice variants were critical for their efficient binding to IL-1RAcP. The synaptogenic activities of IL-1RAcP isoforms were diminished in cortical neurons from PTP $\delta$ knockout mice. Correspondingly, PTP $\delta$ required IL-1RAcPb to induce postsynaptic differentiation. Thus, IL-1RAcPb bidirectionally regulated synapse formation of cortical neurons. Furthermore, the spine densities of cortical and hippocampal pyramidal neurons were reduced in IL-1RAcP knock-out mice lacking both isoforms. These results suggest that IL-1RAcP isoforms function as trans-synaptic cell adhesion molecules in the brain and organize synapse formation. Thus, IL-1RAcP represents an interesting molecular link between immune systems and synapse formation in the brain.

\section{Introduction}

Interleukin-1 (IL-1) family cytokines play important roles in innate and adaptive immune responses and are involved in immune and inflammatory diseases (Dinarello, 2009; Sims and Smith, 2010). The immunological actions of IL-1 family cytokines are mediated by signaling through a group of closely related receptors. The receptors contain extracellular Ig-like domains and a Toll/IL-1 receptor (TIR) domain in the cytoplasmic portion. Binding of each IL-1 family cytokine to its primary receptor subunit initiates the response; in the case of IL-1, IL-1 receptor type I (IL-1R1). The interaction of the cytokine and the ligand-binding subunit allows the recruitment of a second receptor subunit, IL-1R accessory protein (IL-1RAcP). There

\footnotetext{
Received Sept. 12, 2011; revised Nov. 27, 2011; accepted Dec. 28, 2011.

Author contributions: T.Y. and M.M. designed research; T.Y., T.S., S.-J.L., M.Y., and T.U. performed research; Y.I. contributed unpublished reagents/analytic tools; T.Y., T.S., S.-J.L., M.Y., T.U., and X.C. analyzed data; T.Y. and M.M. wrote the paper.

This work was supported in part by research grants from the Ministry of Education, Culture, Sports, Science and Technology of Japan, the Pharmacological Research Foundation, and Japan Science and Technology Agency. We thank M. Watanabe for antibodies against VGluT1, Shank2, and VGAT. We are grateful to T. Shimizu and S. Tokuoka for use of the Biacore 3000 biosensor, and A. Kakihara, R. Suzuki, and A. Maeda for technical assistance.

The authors declare no competing financial interests.

Correspondence should be addressed to Masayoshi Mishina, Department of Molecular Neurobiology and Pharmacology, Graduate School of Medicine, University of Tokyo, Tokyo 113-0033, Japan. E-mail: mishina@m.u-tokyo.ac.jp.

DOI:10.1523/JNEUROSCI.4637-11.2012

Copyright $\odot 2012$ the authors $\quad 0270-6474 / 12 / 322588-13 \$ 15.00 / 0$
}

are five ligand-binding subunits for IL-1 family cytokines, but IL-1RAcP is shared by four of them. Formation of the receptor heterodimer induces signaling because the juxtaposition of the two TIR domains enables the recruitment of signaling intermediates including myeloid differentiation primary response protein 88 (MyD88), IL-1R-associated kinase 4 (IRAK4) and TNFR-associated factor 6 (TRAF6) (O'Neill, 2008). In addition, the IL-1 family cytokines have effects outside of the immune system. In addition to the regulation of neuroinflammation and cell death in neurodegenerative conditions, IL-1 signaling is also involved in sleep regulation, learning, memory, stress responses, and control of lipid metabolism in the brain (Horai et al., 1998; Rothwell and Luheshi, 2000; Matsuki et al., 2003; Krueger, 2008; Goshen and Yirmiya, 2009).

IL-1 receptor accessory protein-like 1 (IL1RAPL1) belongs to the IL-1 receptor family, but has no activity to mediate immune signals as a component of the receptors for IL-1 family cytokines (Born et al., 2000; Sims and Smith, 2010). In contrast to the members of the IL-1 receptor family mediating immune responses to IL-1 family cytokines, IL1RAPL1 is selectively expressed in the brain. IL1RAPL1 was initially identified as the product of an X-linked gene responsible for a nonsyndromic form of mental retardation (MR) (Carrié et al., 1999). We found that presynaptic IL1RAPL1 regulates synapse formation in vivo of olfactory sensory neurons in zebrafish (Yoshida and Mishina, 2008). Recently, we showed that postsynaptic 

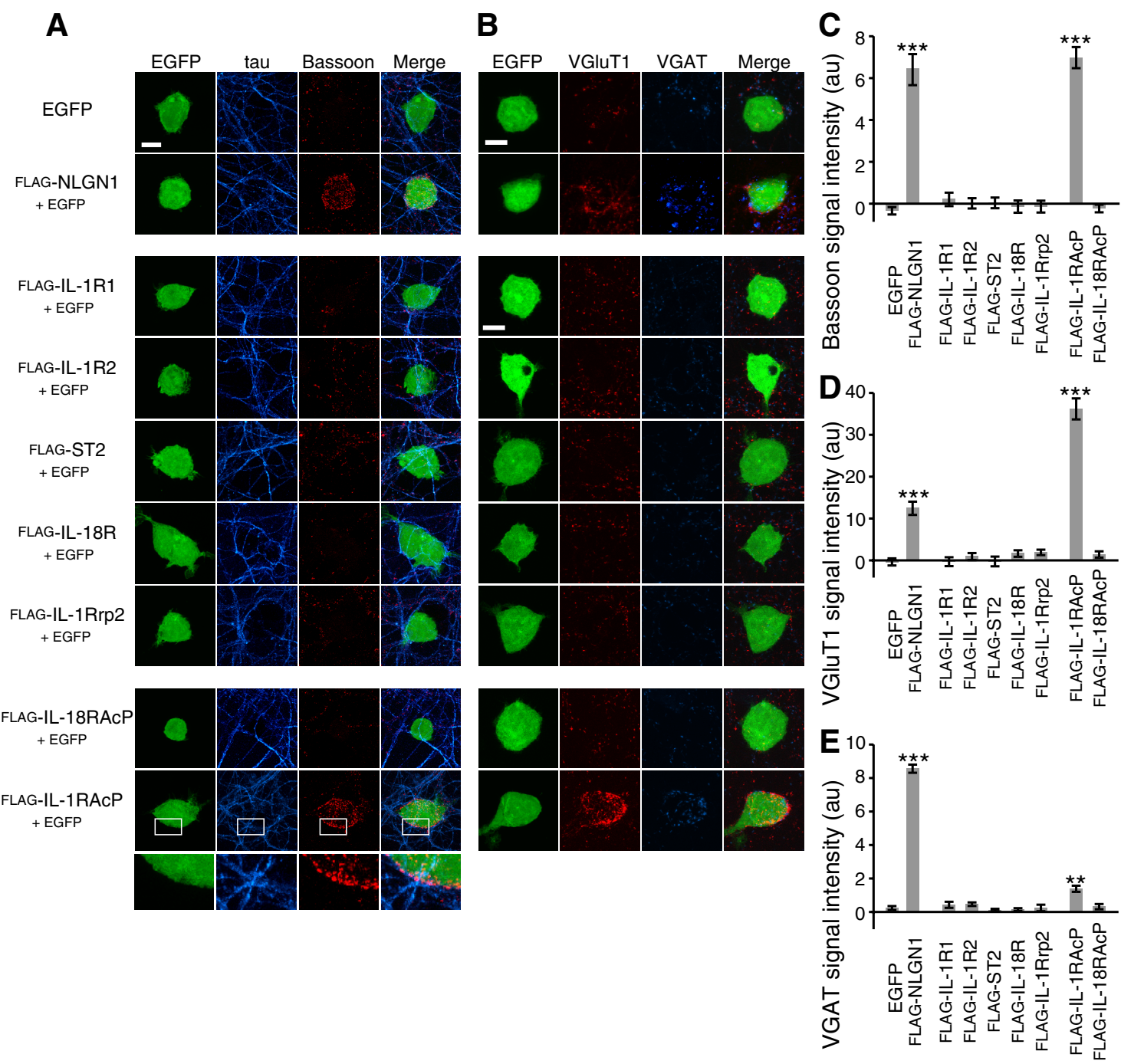

Figure 1. IL-1RACP induces presynaptic differentiation. $\boldsymbol{A}, \boldsymbol{B}$, Cocultures of cortical neurons and HEK293T cells expressing EGFP, FLAG-tagged IL-1R family proteins or FLAG-NLGN1 were immunostained for tau and Bassoon $(\boldsymbol{A})$ and for VGluT1 and VGAT $(\boldsymbol{B})$. For boxed areas in $(\boldsymbol{A})$, higher-magnification images are shown on the bottom. $(-\boldsymbol{E}$, Intensity of staining signals for Bassoon (C), VGluT1 (D), and VGAT (E) on the surface of HEK293T cells expressing EGFP, FLAG-tagged IL-1R family proteins or FLAG-NLGN1 $(n=11-25)$. All values represent mean \pm SEM, ${ }^{* *} p<0.01$ and ${ }^{* * *} p<0.001$ compared with control HEK293T cells expressing EGFP alone; Tukey's test. Scale bars, $10 \mu \mathrm{m}$.

IL1RAPL1 mediates synapse formation of mouse cortical neurons through trans-synaptic interaction with presynaptic protein tyrosine phosphatase (PTP) $\delta$ (Yoshida et al., 2011). Here, we examined the possible role of IL-1R family members in synapse formation in the brain. Screening by mixed culture experiments revealed robust synaptogenic activity of IL-1RAcP in cultured cortical neurons. IL-1RAcP exists in two isoforms designated as IL-1RAcP and IL-1RAcPb, which differ only in the C-terminal region (Smith et al., 2009). The synaptogenic function of IL$1 \mathrm{RAcP}$ isoforms required the interaction with $\mathrm{PTP} \delta$ in a variantselective manner. These results suggest that IL-1RAcP isoforms act as trans-synaptic cell adhesion molecules to organize synapse formation in the brain. Thus, IL-1RAcP provides an interesting molecular link between immune systems and synapse formation in the brain.

\section{Materials and Methods}

Construction of expression vectors. The entire coding sequences of mouse IL-1RAcP, IL-1RAcPb, SynCAM1, SynCAM2, SynCAM3, SALM1, SALM2, SALM3, SALM4 and IL-18RAcP were obtained by reverse transcription (RT)-PCR using mRNA prepared from mouse brains as a tem- plate and cloned into pcDNA3 vector (Invitrogen) or pcDNA3.1D/V5His-TOPO vector (Invitrogen) to yield pIL-1RAcP, pIL-1RAcPb, pSynCAM1, pSynCAM2, pSynCAM3, pSALM1, pSALM2, pSALM3, pSALM4 and pCR-IL-18RAcP, respectively. The expression vectors for neurexin $(\mathrm{NRXN})$ variants and neuroligin (NLGN) 1 were previously described (Uemura et al., 2010; Yoshida et al., 2011). The expression vectors for full-length proteins and ECDs of PTP $\delta$, LAR (leukocyte common antigen-related), and PTP $\sigma$ variants were previously described (Yoshida et al., 2011). The entire coding sequences of mouse IL-1R2, ST2, IL-18R, IL-1Rrp2, IL-18RAcP and IL1RAPL2 were obtained by RT-PCR using mRNA prepared from mouse brains, spleens, or thymuses as templates and cloned into pCRII TOPO vector (Invitrogen) to yield pCR-IL-1R2, pCR-ST2, pCR-IL-18R, pCR-IL-1Rrp2, pCR-IL-18RAcP, and pCRIL1RAPL2, respectively. The coding sequence of preprotrypsin signal peptide followed by FLAG tag was ligated with the coding sequences of IL-1R2, IL-1RAcP, IL-1RAcPb, ST2, IL-18R, IL-1Rrp2, IL-18RAcP, IL1RAPL2 and NLGN1 lacking signal peptide to obtain FLAG-IL-1R2, FLAG-IL1RAcP, FLAG-IL-1RAcPb, FLAG-ST2, FLAG-IL-18R, FLAG-IL-1Rrp2, FLAG-IL-18RAcP, FLAG-IL1RAPL2, and FLAG-NLGN1 coding sequences, respectively. The coding sequences of FLAG-IL-1R2, FLAG-IL1RAcP, FLAG-IL-1RAcPb, FLAG-ST2, FLAG-IL-18R, FLAG-IL-1Rrp2, FLAG-IL-18RAcP, FLAG-IL1RAPL2, and FLAG-NLGN1 were cloned 
into the multicloning site of pEGFP-CAGMCS (Uemura et al., 2010) to yield pEGFP-CAGFLAG-IL-1R2, pEGFP-CAG-FLAG-IL-1RAcP, pEGFP-CAG-FLAG-IL-1RAcPb, pEGFP-CAGFLAG-ST2, pEGFP-CAG-FLAG-IL-18R, pEGFPCAG-FLAG-IL-1Rrp2, pEGFP-CAG-FLAG-IL18RAcP, pEGFP-CAG-FLAG-IL1RAPL2, and pEGFP-CAG-FLAG-NLGN1, respectively. pEGFP-CAG-FLAG-IL-1R1 and pEGFP-CAGFLAG-IL1RAPL1 were previously described (Yoshida et al., 2011). The entire coding sequences of PTP $\delta$, LARA6 and PTP $\sigma \mathrm{A}^{-}$were cloned into the multicloning site of pRFP-CAGMCS (Uemura et al., 2010) to yield pRFP-CAG-PTP $\delta$, pRFP-CAGLARA6 and pRFP-CAG-PTP $\sigma \mathrm{A}^{-}$, respectively. The DNA fragments encoding the ECDs of mouse IL-1R2，IL-1RAcP，ST2，IL-18R，IL-1Rrp2，IL18RAcP, IL1RAPL2 and PTP $\delta A 3$ were amplified by PCR using pCR-IL-1R2, pIL-1RAcP, pCR-ST2, pCR-IL-18R, pCR-IL-1Rrp2, pCR-IL-18RAcP, pCR-IL1RAPL2 and pPTP $\delta A 3$ (Yoshida et al., 2011) as templates, respectively. The amplified fragments were cloned into the XhoI-NotI sites of pEB6-Ig $\kappa$-Fc (Uemura and Mishina, 2008) to yield pEB6-IL-1R2-ECD-Fc, pEB6-IL-1RAcPECD-Fc, pEB6-ST2-ECD-Fc, pEB6-IL-18RECD-Fc, pEB6-IL-1Rrp2-ECD-Fc, pEB6-IL18RAcP-ECD-Fc, pEB6-IL1RAPL2-ECD-Fc and pEB6-PTP $\delta A 3-E C D-F c$. The expression vectors for ECD of IL-1R1 and IL1RAPL1 were previously described (Yoshida et al., 2011).

Animals. PTP $\delta$ knock-out mice were previously generated (Uetani et al., 2000) and IL1RAcP knock-out mice were obtained from Jackson Laboratories. Mice were fed ad libitum with standard laboratory chow and water in standard animal cages under a $12 \mathrm{~h}$ light/dark cycle. Mice of either sex were used for the experiments unless otherwise specified. All animal procedures were approved by the Animal Care and the Use Committee of Graduate School of Medicine, the University of Tokyo (Approval \# 1721T062).

Cell cultures, transfection, and coculture assay. Primary cortical cultures were prepared from mice at postnatal day $0(\mathrm{P} 0)$ essentially as described previously (Uemura et al., 2004). The cortical cells were placed on coverslips coated with $30 \mu \mathrm{g} / \mathrm{ml}$ poly-L-lysine and $10 \mu \mathrm{g} / \mathrm{ml}$ mouse laminin at the density of $2 \times 10^{5}$ cells/well for magnetofection of expression vectors, $3 \times 10^{5}$ cells/well for siRNA-mediated knockdown, and $5 \times 10^{5}$ cells/well for coculture assay. The cells were cultured in Neurobasal-A supplemented with $2 \%$ B-27 supplement (Invitrogen), 5\% fetal calf serum, $100 \mathrm{U} / \mathrm{ml}$ penicillin, $100 \mu \mathrm{g} / \mathrm{ml}$ streptomycin, and $0.5 \mathrm{~mm}$ L-glutamine for $24 \mathrm{~h}$, and then cultured in the same medium without fetal calf serum. Cultures of HEK293T cells were maintained as described previously (Uemura et al., 2004). Expression vectors were transfected to HEK293T cells using FuGene6 transfection reagent (Roche). After $2 \mathrm{~d}$ of culture, the transfected cells were washed with PBS containing 2 mM EDTA, and incubated with the same buffer at $37^{\circ} \mathrm{C}$ for $10 \mathrm{~min}$. Dispersed cells were plated onto cortical neurons at days in vitro (DIV) 14 in the presence or absence of $2 \mu \mathrm{M}$ recombinant proteins. After $24 \mathrm{~h}$ of coculture, cells were fixed for immunostaining. Fc and ECDs of IL-1R family proteins fused to Fc were bound to Protein A-conjugated magnetic particles (smooth surface, $4.0-4.5 \mu \mathrm{m}$ diameter; Spherotech). Conjugated Fc fusion proteins on beads were analyzed by SDS-PAGE followed by immunoblotting with anti-Fc antibody. Beads coupled with Fc or Fc fusion proteins were added to cortical neurons (DIV 8). After $24 \mathrm{~h}$, cultures were fixed for immunostaining. Cultured neurons at DIV 12 were transfected
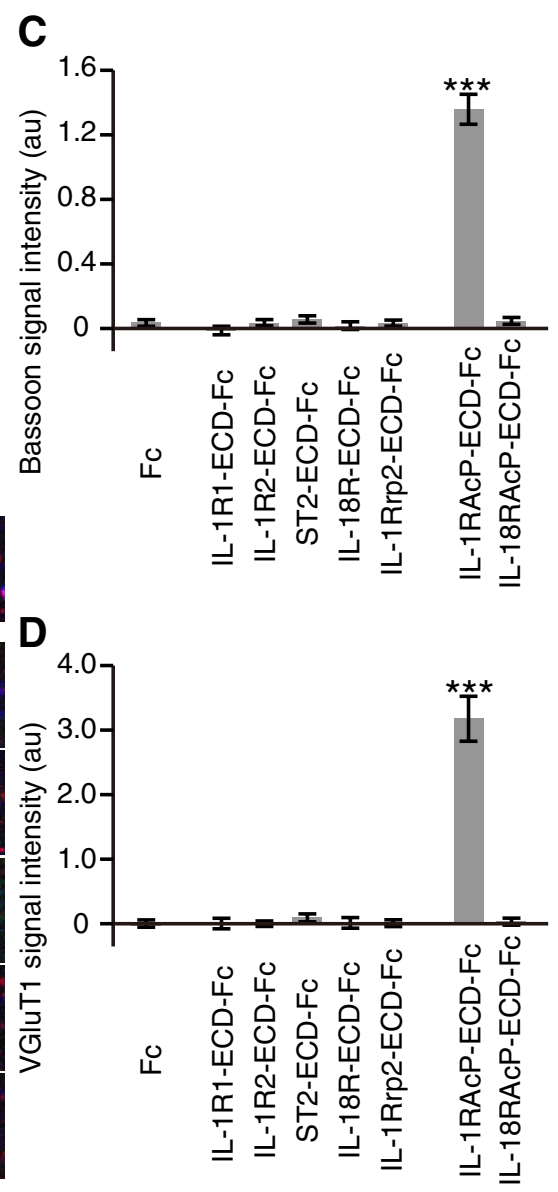

Figure 2. The ECD of IL-1RACP is sufficient to induce presynaptic differentiation. $\boldsymbol{A}$, The amounts of immobilized ECDs of IL-1R family proteins fused with Fc on beads. ECDs of IL-1R family proteins fused with Fc conjugated on beads were separated by SDS-PAGE followed by immunoblotting with anti-Fc $\gamma$ antibody. $\boldsymbol{B}$, Accumulation of Bassoon and VGluT1 signals of cultured cortical VGluT1 (D) on the surface of beads conjugated with Fc or ECDs of IL-1R family proteins fused to FC. $(n=17-21)$. All values represent mean $\pm S E M,{ }^{* * *} p<0.001$ compared with control Fc-coated beads; Tukey's test. Scale bar, $5 \mu \mathrm{m}$.

with expression vectors by magnetofection using NeuroMag transfection reagent (OZ Biosciences) according to the manufacturer's instruction and fixed at DIV 14 for immunostaining.

Small interference RNA-mediated knockdown. An siRNA against Ill rap was custom synthesized (Stealth RNAi, Invitrogen). The sense sequence of siRNA was 5' -CCCAGUGCACAAGAUGUAUAUUGAA- ${ }^{\prime}$. For control siRNA, Stealth RNAi Negative Control kit (Invitrogen) was used. Expression vectors for res-FLAG-IL-1RAcP (pEGFP-CAG-res-FLAGIL-1RAcP) and res-FLAG-IL-1RAcPb (pEGFP-CAG-res-FLAG-IL$1 \mathrm{RAcPb}$ ) were generated by substituting the Ill rap coding sequences 5' -CCCAGTGCACAAGATGTATATTGAA- ${ }^{\prime}$ and $5^{\prime}$-CACTTGGTATAAGGGTTGTACTGAA- $3^{\prime}$ of pEGFP-CAG-FLAG-IL-1RAcP and pEGFP-CAG-FLAG-IL-1RAcPb with 5' -TCCGGTCCATAAAATGTACATCGAG-3' and 5'-GACATGGTACAAAGGATGCACAGAG-3', respectively. Cultured neurons at DIV 10 were transfected with siRNA (20 $\mathrm{pmol} /$ well $)$ and expression vectors $(1 \mu \mathrm{g} /$ well $)$ using NeuroMag transfection reagent and fixed at DIV 14 for immunostaining.

Immunostaining. Immunostaining of primary neuronal cultures and neuron-fibroblast cocultures was performed as described previously (Uemura et al., 2004; Uemura and Mishina, 2008) using chicken 
A

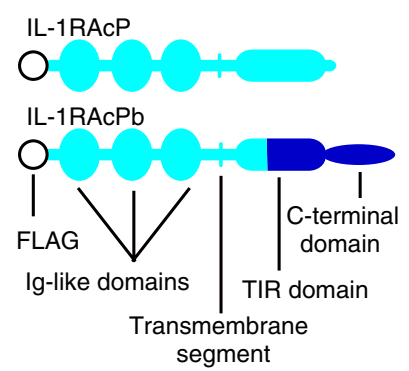

D

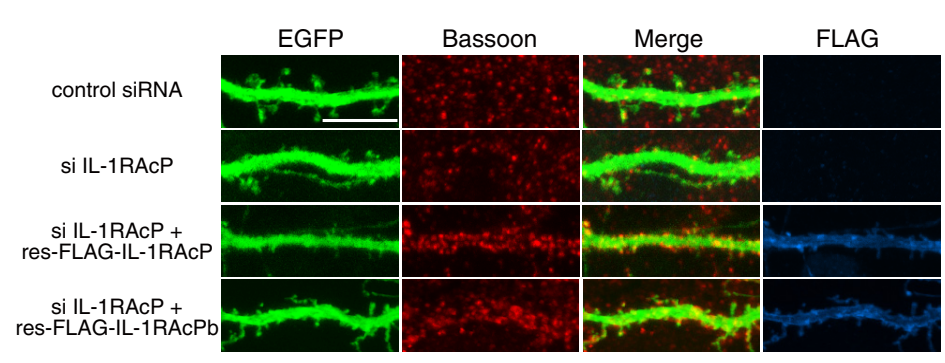

B

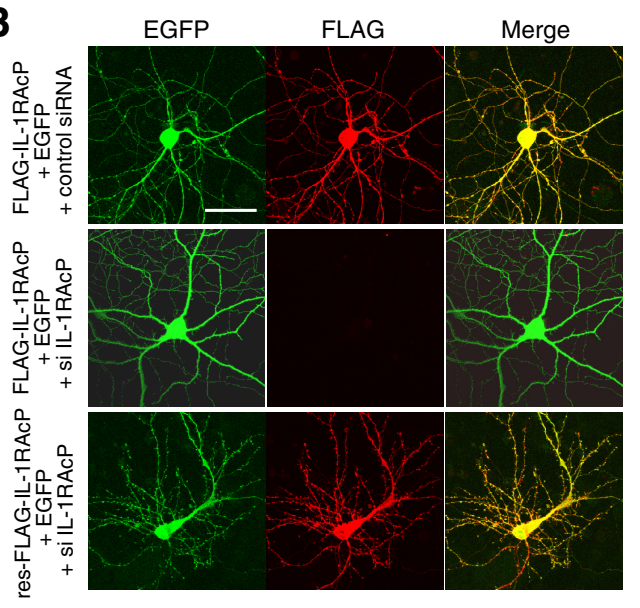

E
C

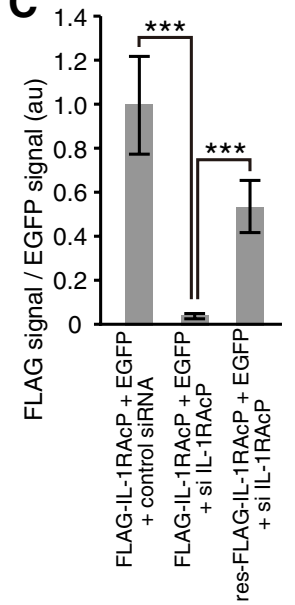

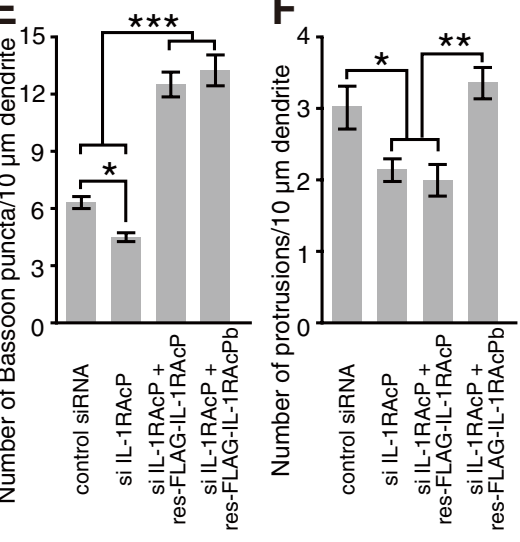

Figure 3. Effect of siRNA-mediated knockdown of IL-1RAcP isoforms on synapse formation. A, Schematic structures of FLAG-IL-1RAcP and FLAG-IL-1RAcPb. B, Cortical neurons were transfected with an siRNA against I/1 rap or control siRNA in combination with expression vectors for EGFP and FLAG-IL-1RAcP or for EGFP and res-FLAG-IL-1RAcP and immunostained for EGFP and FLAG. C, Ratios of FLAG signal intensity and EGFP signal intensity were measured to evaluate the efficacy of siRNA against/11 rap ( $n=11-14)$. $D$, Effects of an siRNA against $/ 17$ rap on accumulation of Bassoon signals along dendrites and dendritic protrusions of cultured cortical neurons and rescue by siRNA-resistant-FLAG-IL-1RAcP (res-FLAG-IL-1RAcP) and siRNA-resistant-FLAG-IL-1RAcPb (res-FLAG-IL1RAcPb). $\boldsymbol{E}, \boldsymbol{F}$, Numbers of Bassoon puncta $(\boldsymbol{E})$ and dendritic protrusions $(\boldsymbol{F})$ along dendrites of cultured cortical neurons treated with siRNAs $(n=23-30)$. All values represent mean $\pm S E M$, ${ }^{*} p<$ $0.05,{ }^{* *} p<0.01$, and ${ }^{* * *} p<0.001$, respectively; Tukey's test. Scale bars: $\boldsymbol{B}, 50 \mu \mathrm{m} ; \boldsymbol{D}, 10 \mu \mathrm{m}$.

anti-FLAG (Kamiya Biomedical Company), rabbit anti-vesicular glutamate transporter 1 (VGluT1) (Miura et al., 2006), mouse antiBassoon (Stressgen), goat anti-vesicular GABA transporter (VGAT) (Miyazaki et al., 2003), goat anti-Tau (Santa Cruz Biotechnology), and rabbit anti-Shank2 (provided by Dr. M. Watanabe, Hokkaido University, Sapporo, Japan) antibodies as primary antibodies. For secondary antibodies, Alexa Fluor 488-, 555-, and 647-conjugated anti-IgG antibodies (Invitrogen) and Cy3- and Cy5-conjugated antiIgG antibodies (Jackson ImmunoResearch) were used. For detection of magnetic beads coated with Fc fusion proteins, FITC-conjugated anti-human Fc $\gamma$ antibody (Jackson ImmunoResearch) was used.

Preparation of soluble recombinant proteins. Soluble recombinant IL1R1-ECD-Fc, IL-1R2-ECD-Fc, IL-1RAcP-ECD-Fc, ST2-ECD-Fc, IL-18RECD-Fc, IL-1Rrp2-ECD-Fc, IL-18RAcP-ECD-Fc, IL1RAPL1-ECD-Fc, IL1RAPL2-ECD-Fc, PTP $\delta$ A3-ECD-Fc, Fc, PTP $\delta$-ECD-MH, PTP $\delta B^{-}{ }_{-}^{-}$

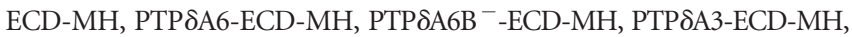
PTP $\delta \mathrm{ABB}^{-}$-ECD-MH, PTP $\delta \mathrm{A}^{-}$-ECD-MH, PTP $\delta \mathrm{A}^{-} \mathrm{B}^{-}$-ECD-MH, LARECD-MH, and PTP $\sigma$-ECD-MH were prepared as described previously (Uemura and Mishina, 2008; Yoshida et al., 2011) by transfection of respective expression vectors into the Freestyle 293 cells (Invitrogen). pAP-tag5 vector was used for AP-MH protein. Fc and Fc fusion proteins were purified from culture media by Protein A-Sepharose (GE Healthcare), eluted with $3 \mathrm{M} \mathrm{MgCl}_{2}$, and dialyzed against HBSS (Invitrogen). AP-MH and ECDs of PTP $\delta$, LAR and PTP $\sigma$ variants tagged with Myc and His epitopes were purified from culture media by Talon metal affinity resin (Clontech) with standard protocol and dialyzed against HBSS.

Cell surface binding assay. Expression vectors for SynCAM1, Syn-
CAM2, SynCAM3, NRXN1 $\beta$, NRXN2 $\beta$, NRXN3 $\beta$, NRXN1 $\alpha$, SALM1, SALM2, SALM3, SALM4 and splice variants of 2A-type RPTPs were cotransfetced with pEGFP-C1 (Clontech) into HEK293T cells. Transfected cells were incubated with either IL-1RAcP-ECD-Fc $(35 \mu \mathrm{g} / \mathrm{ml})$ or $\mathrm{Fc}(15 \mu \mathrm{g} / \mathrm{ml})$ in HBSS containing $2 \mathrm{mM} \mathrm{CaCl}_{2}$ and $1 \mathrm{mM} \mathrm{MgCl}_{2}$ for $1 \mathrm{~h}$ at room temperature. After washing, cells were fixed with $4 \% \mathrm{PFA}$, incubated with rabbit anti-human IgG antibody (Rockland), and immunostained with Alexa Fluor 555-conjugated donkey anti-rabbit IgG antibody (Invitrogen). For the binding between 2A-type RPTPs and IL$1 \mathrm{RAcPb}$, HEK293T cells transfected with pEGFP-CAG-FLAG-IL$1 \mathrm{RAcPb}$ were incubated with AP-MH, PTP $\delta$-ECD-MH, LAR-ECD-MH, or PTP $\sigma$-ECD-MH $(30 \mu \mathrm{g} / \mathrm{ml})$ for $15 \mathrm{~min}$ at room temperature. After washing, cells were fixed with $4 \%$ PFA, incubated with rabbit anti-FLAG (Sigma) and mouse anti-Myc antibodies (Santa Cruz Biotechnology), and immunostained with Alexa Fluor 555-conjugated donkey antimouse IgG and Alexa Fluor 647-conjugated donkey anti-rabbit IgG antibodies (Invitrogen).

Cell aggregation assay. HEK293T cells cotransfected with expression vectors pEGFP-C1 and $\mathrm{pIL}-1 \mathrm{RAcPb}$ and those with ptagRFP-C1 (Evrogen) and $\mathrm{pPTP} \delta$, pLARA 6 , or $\mathrm{PPTP} \sigma \mathrm{A}^{-}$were mixed at a density of $2.0 \times$ $10^{6}$ cells/ml in HBSS containing $2 \mathrm{mM} \mathrm{CaCl}_{2}$ and $1 \mathrm{mM} \mathrm{MgCl}_{2}$. After 20 $\mathrm{min}$, fluorescence images were taken with a fluorescence stereomicroscope (M165 FC, Leica Microsystems).

Surface plasmon resonance binding analysis. Surface plasmon resonance (SPR) binding assay was conducted on a Biacore 3000 biosensor equipped with a sensor chip CM5 (GE Healthcare). Anti-human Fc antibodies were covalently immobilized over independent flow cells using 
Human Antibody Capture Kit (GE Healthcare). IL-1RAcP-ECD-Fc was captured in flow cell 2 or 4 to generate the IL-1RAcP-ECD-Fc sensor chips. Untreated flow cell 1 or 3 served as a reference. Binding analysis was performed at $25^{\circ} \mathrm{C}$ in a running buffer of $10 \mathrm{~mm}$ HEPES, $\mathrm{pH} 7.4,150 \mathrm{~mm} \mathrm{NaCl}, 0.005 \%$ surfactant P20, 2 $\mathrm{mM} \mathrm{CaCl}_{2}$ and $1 \mathrm{~mm} \mathrm{MgCl}$. Analytes in the running buffer were injected in the order of increasing concentrations. Each sample was flowed over the chip surface at a flow rate of 25 $\mu \mathrm{l} / \mathrm{min}$, followed by a dissociation phase. Data analysis was performed using BIAevaluation software Ver. 4.1. The responses were analyzed by steady-state kinetics to calculate the $K_{\mathrm{D}}$.

Preparation of Sindbis viral particles. The coding sequences of FLAG-IL-1RAcP and FLAG-IL-1RAcPb were cloned into pSinRep5 (Invitrogen) to yield pSinRep-FLAG-IL1RAcP and pSinRep-FLAG-IL-1RAcPb, respectively. SP6 RNA polymerase transcripts from these vectors were transfected together with the helper RNA from a defective helper (26S) cDNA template (Invitrogen) into baby hamster kidney cells by electroporation. Twenty-four hours after transfection, the culture medium was harvested to obtain the infectious particles of Sindbis virus. Cultured cortical neurons from Ill rap ${ }^{+/+}$and Ill $\mathrm{rap}^{-/-}$mice were applied with PTP $\delta \mathrm{A} 3-$ ECD-Fc beads and infected with Sindbis viruses for FLAG-IL-1RAcP and FLAG-IL$1 \mathrm{RAcPb}$ at DIV 8 and fixed at DIV 9 for immunostaining.

DiI labeling. DiI labeling was performed essentially as described previously (Kim et al., 2006). Briefly, brains of Il1 $\mathrm{rap}^{+/+}$and Il1 rap ${ }^{-/-}$mice at P20 were fixed with $1.5 \%$ PFA and coronally sectioned into $200 \mu \mathrm{m}$ slices. Solid DiI crystals were applied onto the layer $2 / 3$ of the somatosensory cortex and hippocampal CA1 region. The slices were incubated in PBS at room temperature for $12 \mathrm{~h}$, fixed again with $4 \%$ PFA for $30 \mathrm{~min}$ and washed in PBS three times. Basal dendrites of the pyramidal neurons in the cortical layer $2 / 3$ and hippocampal CA1 were randomly sampled and imaged with a confocal laser-scanning microscope (TCS SP5; Leica, zoom setting; $5, z$ step; $0.17 \mu \mathrm{m}$ ) using Leica $63 \times$ water lens [numerical aperture, 1.20]. Dendritic spines were identified and counted in the 3D projection images. When dendritic spines were too crowded to separate them from each other, we turned to serial stack images to delineate individual spines. By scrolling through the stack of different optical sections, individual spine heads could be identified. All dendritic protrusions with a clearly recognizable neck were counted as spines.

Image acquisition and quantification. Images were collected from at least three separate experiments. Image acquisition and quantification for coculture assays were performed essentially as previously described (Uemura and Mishina, 2008). Briefly, the intensities of immunostaining signals for presynaptic or postsynaptic proteins were measured as the optical mean density within circles of 30 and $7 \mu \mathrm{m}$ diameters enclosing transfected HEK293T cells and coated beads, respectively. For the quantification of numbers of dendritic protrusions and Bassoon puncta, $z$-series optical sections of cortical neurons were projected by the brightest point method using ImageJ $1.36 \mathrm{~b}$ software and the thickest dendrite of a pyramidal cell-like neuron with a recognizable axon was chosen for measurements. Numbers of dendritic protrusions ranging from 0.5 to 8 $\mu \mathrm{m}$ (Takahashi et al., 2003) and Bassoon puncta on the surface of enhanced green fluorescent protein (EGFP)-labeled dendritic shafts and protrusions were counted manually in a blind manner with respect to expression vectors transfected. The average numbers of dendritic protrusions and Bassoon puncta per unit length of dendrites were calculated as the densities. Statistical significance was evaluated by Student's $t$ test or one-way ANOVA followed by post hoc Tukey's test.

\section{Results}

Screening of IL-1R family proteins for synaptogenic activity IL-1R1, IL-1R2, ST2, IL-18R and IL-1Rrp2 are the ligandbinding subunits of the IL-1R family, while IL-1RAcP and IL18RAcP are accessory proteins for the ligand-binding subunits (Sims and Smith, 2010). We examined IL-1R family proteins for their abilities to induce presynaptic differentiation by fibroblastneuron mixed culture assay (Scheiffele et al., 2000). HEK293T cells were transfected with EGFP and respective IL-1R family proteins tagged with FLAG epitope at the $\mathrm{N}$ terminus and were seeded on the top of cultured cortical neurons. HEK293T cells transfected with EGFP and FLAG tagged-NLGN1 and those with EGFP alone served as positive and negative controls, respectively. After $24 \mathrm{~h}$ of coculture, neurons were immunostained for axonal protein tau, active zone protein Bassoon, excitatory presynaptic protein VGluT1 and inhibitory presynaptic protein VGAT (Fig. $1 A, B)$. Little staining signals for Bassoon, VGluT1 and VGAT were detectable on the axons crossing the surface of HEK293T cells expressing ligand-binding subunits of the IL-1R family proteins. On the other hand, we detected robust staining signals for Bassoon on the axons in contact with HEK293T cells expressing FLAG-IL-1RAcP (Fig. 1 A,C). The intensity of staining signals for Bassoon on the axons in contact to the surface of HEK293T cells expressing FLAG-IL-1RAcP was comparable to that on the axons in contact to the surface of HEK293T cells expressing FLAGNLGN1 ( $p=0.998)$. In parallel, HEK293T cells expressing FLAG-IL-1RAcP showed stronger staining signals for VGluT1 than those expressing FLAG-NLGN1 $(p<0.001)$ (Fig. $1 B, D)$. In contrast, little staining signals for Bassoon, VGluT1 and VGAT were detectable on the axons in contact to the surface of HEK293T cells expressing FLAG-IL-18RAcP. These results revealed the activity of IL-1RAcP to induce presynaptic differenti- 
ation of cortical neurons. The synaptogenic activity is unique for IL-1RAcP among IL-1 receptor family members mediating immune signals.

On the other hand, staining signals for VGAT on the axons in contact to the surface of HEK293T cells expressing FLAG-IL1RAcP were much weaker than those on the axons in contact to the surface of HEK293T cells expressing FLAG-NLGN1 $(p<$ 0.001 ) (Fig. $1 B, E$ ). Other members of IL-1R family proteins showed marginal signals. These results suggest that IL-1RAcP has a strong synaptogenic activity to induce presynaptic differentiation and its synaptogenic activity is preferential for excitatory synapses compared with NLGN1.

We next produced and purified the ECD of IL-1R family proteins fused to the Ig constant region $(\mathrm{Fc})$. These fusion proteins were coated on magnetic beads. Immunoblot analysis confirmed that the amounts of immobilized ECD of IL-1R family proteins on beads were comparable (Fig. $2 \mathrm{~A}$ ). These beads were applied to cultured cortical neurons. After incubation for $24 \mathrm{~h}$, cultures were immunostained with antibodies against Bassoon, VGluT1 and human $\mathrm{Fc} \gamma$. We detected numerous punctate staining signals for Bassoon and VGluT1 on the surface of IL-1RAcP-ECD-Fccoated beads (Fig. 2 B). The intensities of staining signals for Bassoon and VGluT1 were significantly stronger than those on Fc-coated control beads $(p<0.001$ ) (Fig. $2 C, D)$. There were no significant signals on the surface of beads coated with ECD of other IL-1R family proteins. These results suggest that the ECD of IL-1RAcP is sufficient to induce presynaptic differentiation.

\section{Neuronal isoform of IL-1RAcP stimulates the accumulation of active zone protein Bassoon and increases the number of dendritic protrusions in cultured cortical neurons}

We thus focused on IL-1RAcP among IL-1R family proteins in subsequent studies. IL-1RAcP exists in two isoforms designated as IL-1RAcP and IL-1RAcPb (Smith et al., 2009). IL-1RAcP is expressed in numerous tissues including brain (Greenfeder et al., 1995), whereas the expression of IL-1RAcPb is restricted in the CNS (Smith et al., 2009). Furthermore, IL-1RAcPb is predominantly expressed in neurons, while IL-1RAcP is found in both neurons and glial cells. Two isoforms share the same N-terminal ECD and differ only in the C-terminal region (Smith et al., 2009) (Fig. 3A).

We examined the effect of siRNA-mediated knockdown of endogenous IL-1RAcP and IL-1RAcPb on synapse formation of cultured cortical neurons. The efficacy of an siRNA directed against common sequence between mouse Il1 rap splice variants was tested by cotransfection with the FLAG-IL-1RAcP expression vector into cortical neurons (Fig. $3 B, C$ ). The siRNA against Ill rap was transfected into cultured cortical neurons at DIV 10. After $4 \mathrm{~d}$ of incubation, we observed a significant reduction of staining signals for presynaptic Bassoon along the dendrites of the transfected cortical neurons and a decrease of number of dendritic protrusions (Fig. $3 D-F)$. The effects of the siRNA on the accumulation of Bassoon puncta and on the number of dendritic protrusions were canceled out by transfection of an siRNA-resistant form of FLAG-IL-1RAcPb (res-FLAG-IL-1RAcPb). On the other hand, an siRNA-resistant form of FLAG-IL-1RAcP (res-FLAG-IL-1RAcP) rescued the accumulation of Bassoon puncta but not the number of dendritic protrusions from siRNA treatment. These results suggest that IL1RAcP has an ability to induce presynaptic differentiation and IL-1RAcPb controls both presynaptic differentiation and spinogenesis of cortical neurons.

We then examined the effects of expression of IL-1RAcP and IL-1RAcPb in cultured cortical neurons on synapse for-

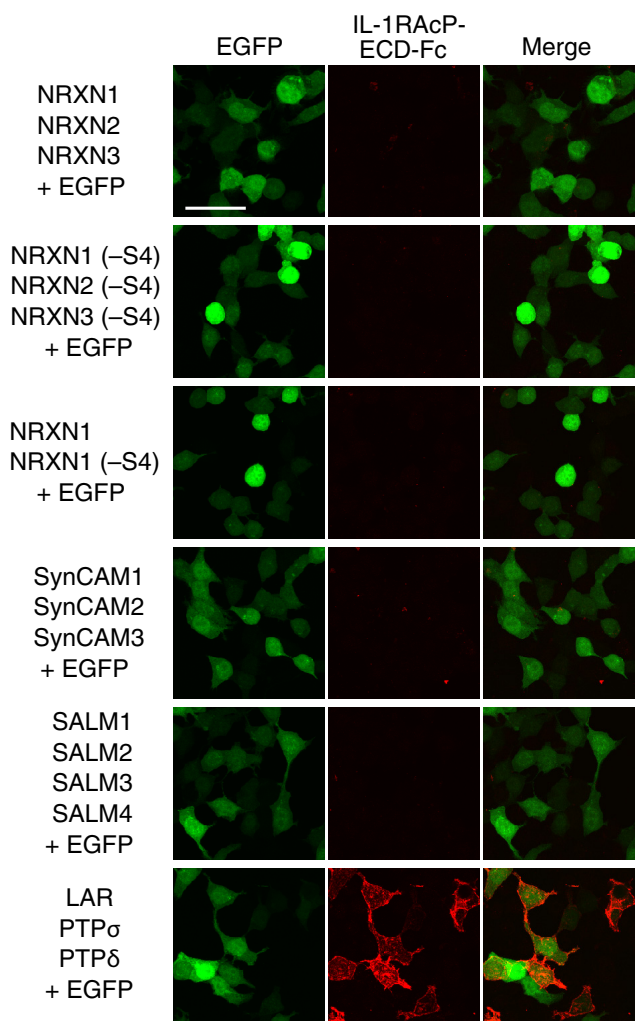

Figure 5. Screening of synaptic cell adhesion molecules for interacting with IL-1RACP-ECD by cell surface binding assay. HEK293T cells transfected with expression vectors for NRXN1 $\beta$, NRXN2 $\beta$, NRXN3 $\beta$ and EGFP, with those for NRXN1 $\beta(-S 4), N R X N 2 \beta(-S 4)$, NRXN3 $\beta(-S 4)$ and EGFP, with those for NRXN1 $\alpha$, NRXN1 $\alpha(-$ S4) and EGFP, with those for SynCAM1, SynCAM2, SynCAM3 and EGFP, with those for SALM1, SALM2, SALM3, SALM4 and EGFP, and with those for LAR, PTP $\sigma$, PTP $\delta$ and EGFP were incubated with IL-1RAcP-ECD-Fc. LAR and PTP $\sigma$ used in the cell surface binding assay are the splice variant containing 6 aa peptide in stead of meA peptide (LARA6) and that lacking meA (PTP $\sigma \mathrm{A}^{-}$), respectively. The transfected cells were immunostained with anti-Fc antibody. Scale bar, $50 \mu \mathrm{m}$.

mation. Transfection of cultured cortical neurons at DIV 12 with the expression vector for FLAG-IL-1RAcP increased punctate immunostaining signals for Bassoon but exerted little effect on dendritic protrusions (Fig. 4). On the other hand, transfection of FLAG-IL-1RAcPb stimulated both the accumulation of Bassoon signals along the dendrites and formation of dendritic protrusions (Fig. 4). These results suggest that both IL-1RAcP and IL-1RAcPb have the ability to induce presynaptic differentiation, while the spinogenic activity is specific for IL-1RAcPb.

\section{IL-1RAcP isoforms interact with PTP $\delta$}

We assume that IL-1RAcP and IL-1RAcPb may exert the synaptogenic activity by interacting with presynaptic protein(s) since the ECD shared by two IL-1RAcP isoforms is sufficient to induce presynaptic differentiation. To examine the issue, we screened presynaptic cell adhesion molecules such as NRXNs, SynCAMs, SALMs and 2A-type receptor-like PTP (RPTP) subfamily for the ability to interact with IL-1RAcP-ECD by cell surface binding assay (Fig. 5). Among them, we detected strong binding signals of IL-1RAcP-ECD-Fc on the surface of HEK293T cells transfected with a mixture of expression vectors for LAR, PTP $\delta$, and PTP $\sigma$. We detected strong binding signals of the ECD of PTP $\delta$ tagged with $\mathrm{Myc}$ and His epitopes at the C-terminus (PTP $\delta$-ECD-MH) on the surface of HEK293T cells expressing IL-1RAcPb, while LAR-ECD-MH and PTP $\sigma$-ECD-MH showed only weak binding 
A

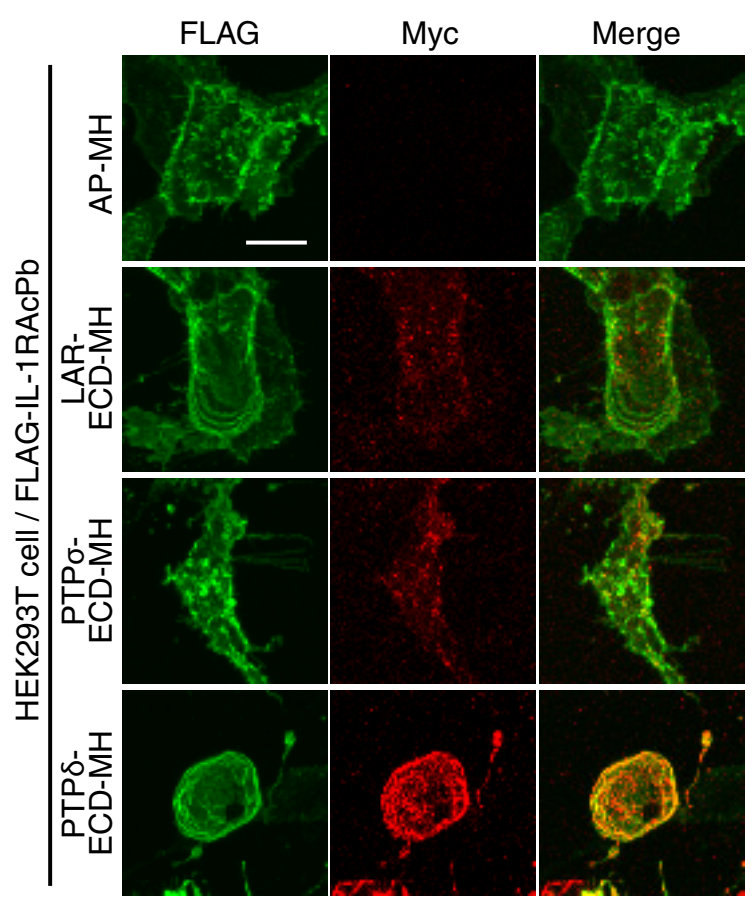

B

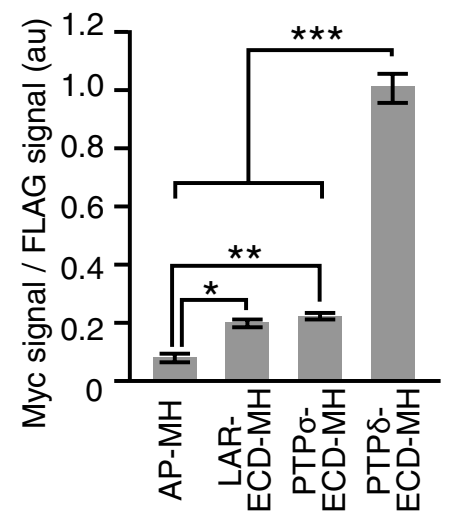

D

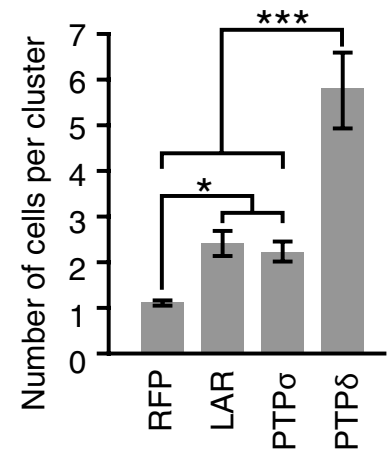

C

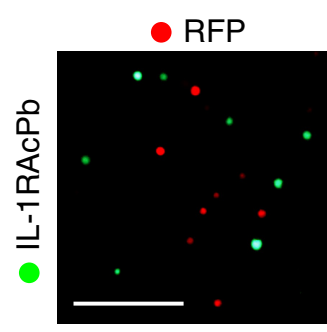

- LAR

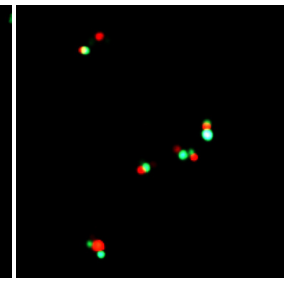

- PTPo

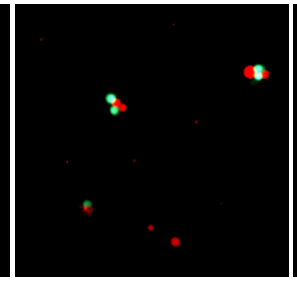

- PTP $\delta$

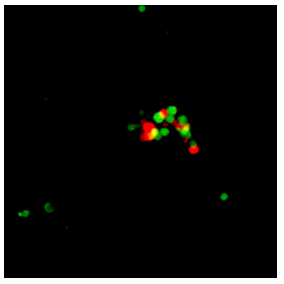

$\mathbf{E}$

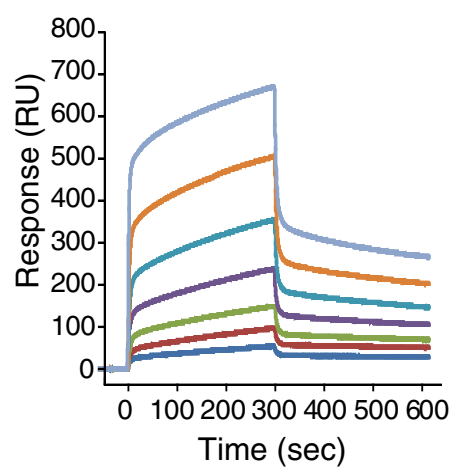

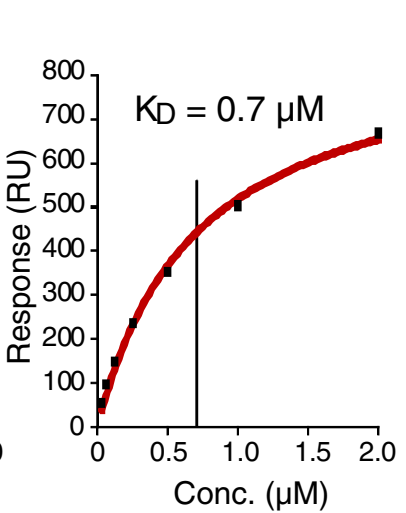

$\mathbf{F}$

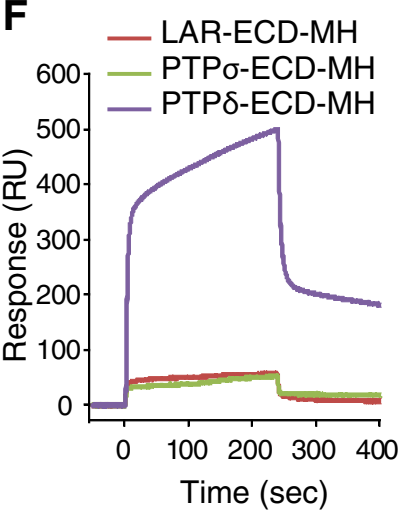

Figure 6. Interaction between IL-1RACP isoforms and PTP $\delta$. A, Binding of LAR-ECD-MH, PTP $\sigma$-ECD-MH and PTP $\delta$-ECD-MH to HEK293T cells transfected with FLAG-IL-1RAcPb. Alkaline phosphatase tagged with Myc and His epitopes (AP-MH) was used as a negative control. $\boldsymbol{B}$, Ratios of cell surface signals for LAR-ECD-MH, PTP $\sigma$-ECD-MH and PTP $\delta$-ECD-MH and those for FLAG-IL-1RAcPb in $\boldsymbol{A}(n=12$ each). C, Cell aggregation assay of HEK293T cells transfected with EGFP and IL-1RAcPb (green) and those with RFP and LAR, PTP $\sigma$ or PTP $\delta$ (red). D, Quantification of cell aggregates in C. E, SPR analysis. Interaction kinetics were measured by passing various concentrations $(31.3,62.5,125,250,500,1000,2000 \mathrm{~nm})$ of purified PTP $\delta$-ECD-MH over IL-1RAcP-ECD-FC captured on the surface of a sensor chip (left). Steady-state kinetics of interaction between PTP $\delta$-ECD-MH and IL-1RACP-ECD-Fc (right). $F$, Interaction kinetics were measured by passing $500 \mathrm{~nm}$ purified LAR-ECD-MH, PTP $\sigma$-ECD-MH or PTP $\delta$-ECD-MH over IL-1RACP-ECD-Fc captured on the surface of a sensor chip. LAR and PTP $\sigma$ used in the cell surface binding assay, cell aggregation assay, and SPR binding analysis are the splice variant containing a 6 a peptide instead of meA peptide (LARA6) and that lacking meA (PTP $\sigma \mathrm{A}^{-}$), respectively. All values represent mean \pm SEM, ${ }^{*} p<0.05,{ }^{* *} p<0.01$, and ${ }^{* * *} p<0.001$, respectively; Tukey's test. Scale bars: $A, 10 \mu \mathrm{m} ; C, 200 \mu \mathrm{m}$. 
A

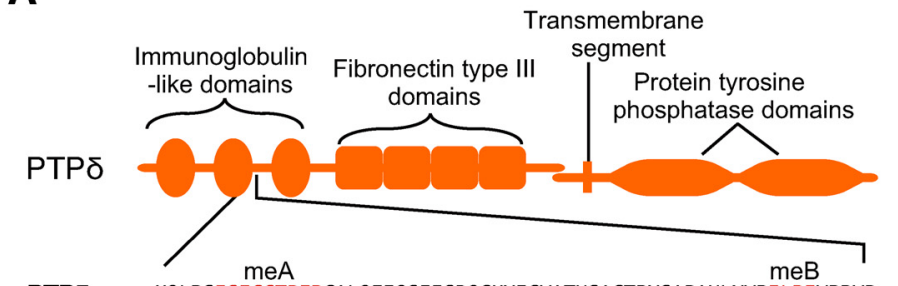

PTPס KQLRSESIGGTPIRGALQIEQSEESDQGKYECVATNSAGTRYSAPANLYVRELREVRRVP

PTPסB ${ }^{-} \quad$ KQLRSESIGGTPIRGALQIEQSEESDQGKYECVATNSAGTRYSAPANLYVR----VRRVP

PTPסA6 KQLRS---GGTPIRGALQIEQSEESDQGKYECVATNSAGTRYSAPANLYVRELREVRRVP

PTPסA6B ${ }^{-}$KQLRS---GGTPIRGALQIEQSEESDQGKYECVATNSAGTRYSAPANLYVR----VRRVP

PTPסA3 KQLRSESI------GALQIEQSEESDQGKYECVATNSAGTRYSAPANLYVRELREVRRVP

PTPJA3B- KQLRSESI------GALQIEQSEESDQGKYECVATNSAGTRYSAPANLYVR----VRRVP

PTPסA ${ }^{-}$KQLRS-------GALQIEQSEESDQGKYECVATNSAGTRYSAPANLYVRELREVRRVP

PTPסA-B- KQLRS--------GALQIEQSEESDQGKYECVATNSAGTRYSAPANLYVR----VRRVP

C

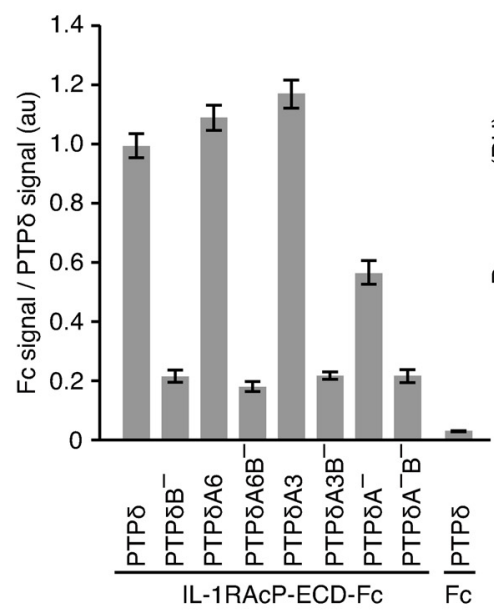

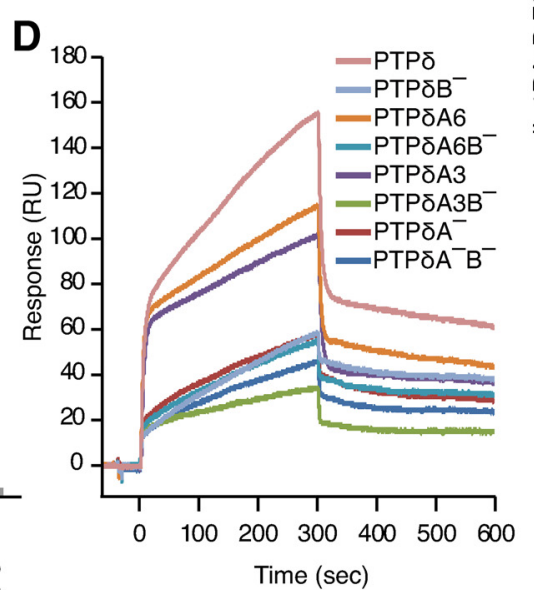

B

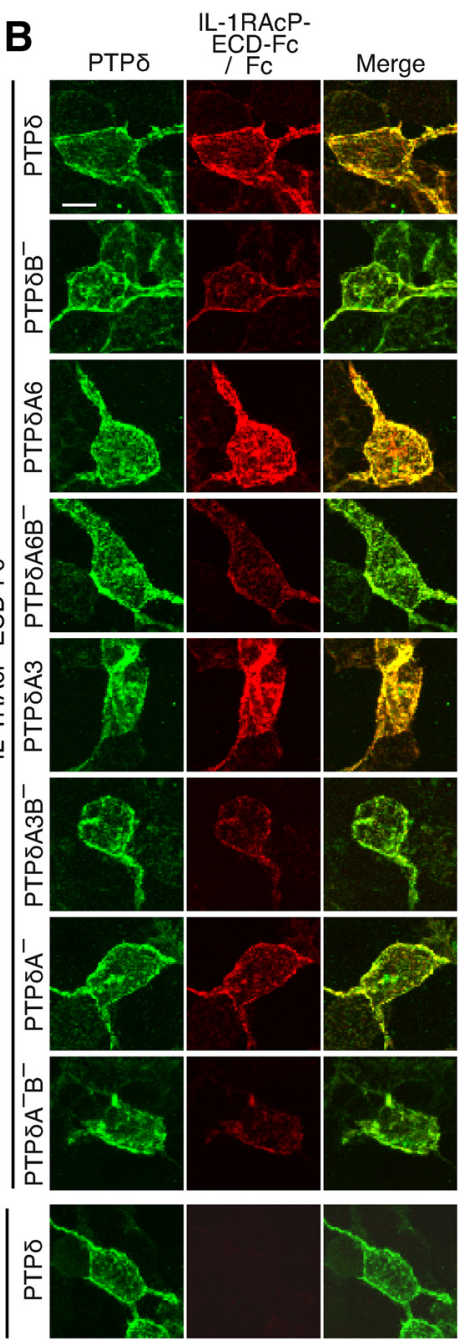

Figure 7. Effects of mini-exon peptides of PTP $\delta$ on the interaction with IL-1RAcP isoforms. $A$, Schematic structure of PTP $\delta$ and amino acid sequences of splice variants in Ig-like domains. Variants expressed in brains of mice at P11 are indicated in bold. B, Binding of IL-1RAcP-ECD-Fc to HEK293T cells transfected with PTP $\delta$ splice variants. C, Ratios of staining signals for IL-1RACP-ECD-Fc and PTP $\delta$ splice variants in $(\boldsymbol{B})\left(n=12\right.$ each). D, SPR analysis. Interaction kinetics were measured by passing $100 \mathrm{~nm}$ purified PTP $\delta$-ECD-MH, PTP $\delta B^{-}{ }^{-}$-ECD-MH, PTP $\delta A 6-E C D-M H, P T P \delta A 6 B^{-}-{ }^{-}$ECD-MH, PTP $\delta A 3-E C D-M H, P T P \delta A 3 B^{-}-E C D-M H, P T P \delta A^{-}-E C D-M H$ and PTP $\delta A^{-} B^{-}-E C D-M H$ over IL-1RAcP-ECD-Fc captured on the surface of a sensor chip. All values represent mean \pm SEM. Scale bar, $10 \mu \mathrm{m}$.

signals (Fig. 6A,B). To examine whether the heterophilic binding between IL-1RAcP-ECD and PTP $\delta$-ECD mediates cell adhesion, we incubated HEK293T cells transfected with IL$1 \mathrm{RAcPb}$ and EGFP and those transfected with PTP $\delta$ and RFP (Fig. 6C,D). The transfected cells aggregated into large clumps, indicating that IL- $1 \mathrm{RAcPb}$ and $\mathrm{PTP} \delta$ function as heterophilic cell adhesion molecules. Smaller aggregates were formed between HEK293T cells transfected with LAR or PTP $\sigma$ and those transfected with IL-1RAcPb. None of the HEK293T cells expressing IL-1RAcPb, PTP $\delta$, LAR or PTP $\sigma$ showed homophilic aggregation.

To quantify the interaction between IL-1RAcP isoforms and PTP $\delta$, we used SPR binding assay. Increasing concentrations of PTP $\delta$-ECD-MH were injected over IL-1RAcP-ECD-Fc-tethered chip surface (Fig. $6 E$ ). Analysis of the steady-state kinetics of the sensorgrams showed a dissociation constant $\left(K_{\mathrm{D}}\right)$ of $0.7 \mu \mathrm{M}$, indicating a high affinity interaction between IL-1RAcP isoforms and PTP $\delta$. The amounts of LAR-ECD-MH or PTP $\sigma$-ECD-MH bound to IL-1RAcP-ECD were much smaller than those of PTP $\delta$ ECD-MH (Fig. 6F).

\section{Mini-exon peptides in PTP $\delta$ are important for binding to}

\section{IL-1RAcP isoforms}

Splice variants with or without mini-exon (me) peptides in Iglike domains were reported for human and mouse PTP $\delta$ (Pulido et al., 1995; Yoshida et al., 2011). Among 8 possible splice variants shown in Figure $7 A$, at least 6 variants (PTP $\delta, \mathrm{PTP} \delta A 6, \mathrm{PTP} \delta \mathrm{A} 3$, $\mathrm{PTP} \delta \mathrm{A}_{3} \mathrm{~B}^{-}, \mathrm{PTP} \delta \mathrm{A}^{-}$and $\mathrm{PTP} \delta \mathrm{A}^{-} \mathrm{B}^{-}$) were expressed in the developing mouse brain at P11 (Yoshida et al., 2011). We expressed 8 possible splice variants of PTP $\delta$ in HEK293T cells and examined their binding ability to IL-1RAcP isoforms. Strong staining signals for IL-1RAcP-ECD-Fc were detected on the surface of HEK293T cells expressing PTP $\delta, \mathrm{PTP} \delta \mathrm{A} 6$ or PTP $\delta$ A 3 and moderate staining signals for IL-1RAcP-ECD-Fc were detected on the surface of HEK293T cells expressing PTP $\delta A^{-}$(Fig. 7 B, C). On the other hand, only weak signals for IL-1RAcP-ECD-Fc were detectable on the surface of HEK293T cells expressing PTP $\delta$ variants lacking the meB peptide $\left(\mathrm{PTP} \delta \mathrm{B}^{-}, \mathrm{PTP} \delta \mathrm{A}^{-} \mathrm{B}^{-}, \mathrm{PTP} \delta \mathrm{A} 3 \mathrm{~B}^{-}\right.$ and PTP $\delta \mathrm{A}^{-} \mathrm{B}^{-}$) (Fig. $\left.7 \mathrm{~B}, \mathrm{C}\right)$. Correspondingly, SPR binding analysis revealed the amounts of PTP $\delta$-ECD-MH, PTP $\delta A 6-$ ECD-MH and PTP $\delta A 3-E C D-M H$ bound to IL-1RAcP-ECD 
were much larger than those of other PTP $\delta$ variants (Fig. 7D). These results suggest that the insertion of the meB peptide into the Ig-like domains is essential for binding to IL-1RAcP. In addition, the 6- or 3 aa meA peptide is important for efficient binding.

PTP $\delta, P T P \sigma$ and LAR share similar domain structures and constitute 2A-type RPTP (Tonks, 2006). In the developing mouse brain, predominant variants of PTP $\sigma$ are lacking meA peptide and those of LAR are without meB peptide. These PTP $\sigma$ and LAR variants expressed in the brain showed significant binding signals for IL-1RAcP-ECD (data not shown). Similarly to $\mathrm{PTP} \delta$, LAR and PTP $\sigma$ variants with mini-exon peptides showed stronger binding activities to IL-1RAcPECD than those without mini-exon peptides.

\section{IL-1RAcP isoforms require PTP $\delta$ for induction of presynaptic differentiation} To examine whether IL-1RAcP isoforms require $\mathrm{PTP} \delta$ for their synaptogenic activities, HEK293T cells transfected with FLAG-IL-1RAcPb were cocultured with cortical neurons prepared from $\mathrm{PTP} \delta$ knock-out mice (Uetani et al., 2000). Then, the cocultures were immunostained with antibody against Bassoon. We detected numerous punctate staining signals for Bassoon on the surface of HEK293T cells expressing FLAG-IL-1RAcPb when cocultured with cortical neurons from wild-type mice (Fig. 8). On the other hand, the Bassoon signals on the HEK293T cells expressing FLAG-IL-1RAcPb were markedly decreased in the cocultures with cortical neurons prepared from PTP $\delta$ knock-out mice. Similar results were obtained for HEK293T cells expressing FLAG-IL-1RAcP (data not shown). These results suggest that IL-1RAcP isoforms require $\mathrm{PTP} \delta$ for the induction of presynaptic differentiation. The remaining Bassoon signals may be ascribed to possible interactions between IL-1RAcP and PTP $\sigma$ or LAR.

\section{PTP $\delta$ induces postsynaptic differentiation through interaction with IL-1RAcPb}

Since the expression of IL-1RAcPb increased the number of dendritic protrusions in cortical neurons, we further examined whether the interaction between IL-1RAcP isoforms and PTP $\delta$ affects the postsynaptic differentiation. Magnetic beads conjugated with the ECD of PTP $\delta A 3$ which showed strong binding activity to IL-1RAcP isoforms (Fig. 7C) were applied to cultured cortical neurons from

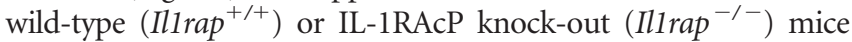
(Cullinan et al., 1998). The Ill rap-targeted deletion eliminates two exons in the extracellular domain and thus destroys the expression of all functional IL-1RAcP isoforms (Cullinan et al., 1998). Immunostaining for excitatory postsynaptic protein Shank2 revealed PTP $\delta A 3-E C D-F c-c o a t e d$ beads showed a robust activity to induce excitatory postsynaptic differentiation in cortical neurons from wild-type mice (Fig. 9A,B). On the other hand, Shank2 signals on the PTP $\delta$ A3-ECD-Fc-coated beads were markedly decreased in the cortical neurons prepared from IL-1RAcP knock-out mice. These results suggest that $\mathrm{PTP} \delta \mathrm{A} 3$ requires IL-1RAcP isoforms for the induction of postsynaptic differentiation. The Sindbis virus-mediated expression of FLAG-IL-1RAcPb recovered Shank2 staining signals on PTPSA3-ECD-Fc-coated beads while the expression of FLAGIL-1RAcP failed to restore staining signals for Shank2 on the beads though the beads were surrounded by the FLAG-IL-1RAcP expressing dendrites (Fig. 9C,D). These results suggest that PTP $\delta$ induces postsynaptic differentiation through interaction with IL-1RAcPb.

\section{Ablation of IL-1RAcP isoforms reduces spine densities of cortical and hippocampal neurons in vivo}

Since IL-1RAcP isoforms regulate synapse formation in cultured cortical neurons, we finally examined whether IL-1RAcP isoforms are involved in synapse formation in vivo in the developing mouse brain. It is reported that both IL-1RAcP isoforms and PTP $\delta$ are widely expressed in the mouse brain including cerebral cortex and hippocampus (Smith et al., 2009; Kwon et al., 2010). Thus, we labeled cerebral cortical and hippocampal neurons of wild-type and IL-1RAcP knock-out mice with DiI and found the decrease in spine densities of basal dendrites of cortical layer $2 / 3$ and hippocampal CA1 pyramidal neurons in IL-1RAcP knockout mice at P20 ( $p<0.05$ and $<0.01$, respectively) (Fig. 10). These results suggest that IL-1RAcP isoforms are involved in the excitatory synapse formation of cortical and hippocampal neurons in vivo.

\section{Discussion}

IL-1 family cytokines play critical roles in immune regulation and inflammation (Dinarello, 2009; Sims and Smith, 2010). IL$1 \mathrm{RAcP}$ is the essential component of the signaling-competent receptor complexes for IL-1 $\alpha$, IL-1 $\beta$, IL-33, IL-1F6, IL-1F8 and IL-1F9. Among IL-1 receptor family proteins, IL-1R1, ST2 and IL-1Rrp2 use IL-1RAcP as the coreceptor (Dinarello, 2009; Sims and Smith, 2010). In the brain, IL-1R1 and IL-1RAcP are expressed in both neuronal and glial cells (Yabuuchi et al., 1994; Ericsson et al., 1995; Friedman, 2001; Smith et al., 2009), while $\mathrm{IL}-1 \mathrm{RAcPb}$ is predominantly expressed in neuronal cells (Smith et al., 2009). ST2 and IL-1Rrp2 are predominantly expressed in glial cells (Andre et al., 2005). IL-1 in the brain mediates host defense responses to neuroinflammation and cell death in neurodegenerative conditions and also acts as an endogenous pyro- 
A
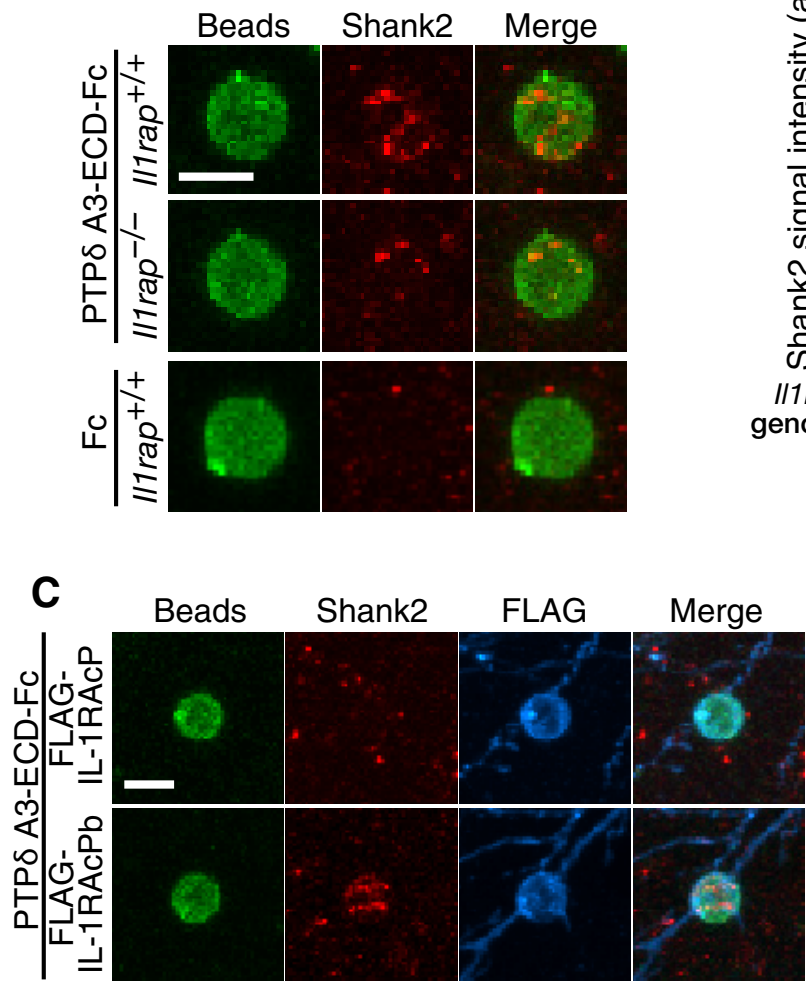

B

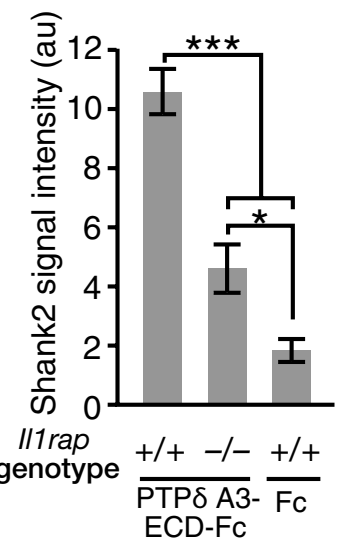

D

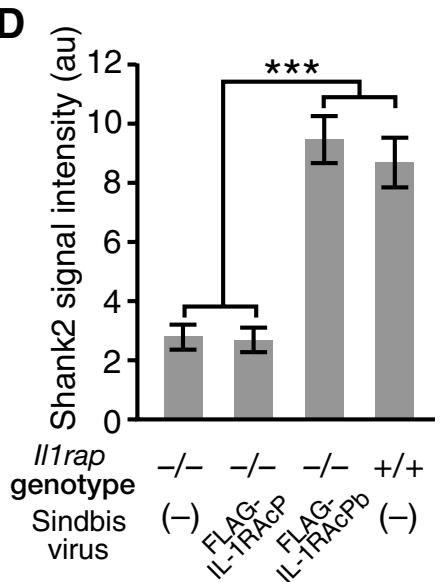

Figure 9. PTP $\delta$ induces postsynaptic differentiation through IL-1RAcPb. $A$, Accumulation of Shank2 signals of cultured cortical neurons from wild-type $\left(\right.$ (II1 rap $\left.{ }^{+/+}\right)$and IL-1RAcP knock-out $\left(/ 17 \mathrm{rap}^{-/-}\right)$mice by beads conjugated with PTP $\delta$ A3-ECD-Fc or Fc protein. $\boldsymbol{B}$, Intensity of staining signals for Shank2 on the surface of beads conjugated with PTP $\delta A 3-E C D-F c$ or Fc. $(n=47$ each).

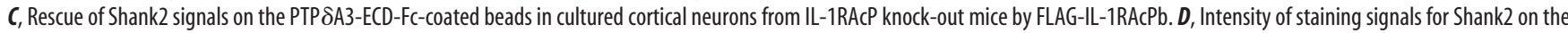
surface of beads conjugated with PTP $\delta$ A3-ECD-Fc in cultured cortical neurons from IL-1RACP knock-out mice infected with Sindbis viruses for FLAG-IL-1RACP and FLAG-IL-1RACPb ( $n=30$ each). Staining signals for Shank2 in noninfected neurons from wild-type and IL-1RAcP knock-out mice served as positive and negative controls, respectively ( $n=15$ and 30 , respectively). All values represent mean $\pm \mathrm{SEM}^{*} p<0.05$ and ${ }^{* * *} p<0.001$, respectively; Tukey's test. Scale bar, $5 \mu \mathrm{m}$.

gen (Dascombe, 1985; Rothwell and Luheshi, 2000; Dinarello, 2009). Correspondingly, IL-1RAcP is involved in the development of experimental autoimmune encephalomyelitis while IL$1 \mathrm{RAcPb}$ controls neuronal vulnerability to local LPS challenge in the CNS (Smith et al., 2009). It was reported that IL-1R1 knockout mice display slower rate of learning in the spatial memory paradigm and impaired short-term and long-term plasticity in the hippocampus (Avital et al., 2003). On the other hand, administration of IL-1 produces detrimental effects on learning and memory processes (Oitzl et al., 1993; Gibertini et al., 1995; Aubert et al., 1995; Pugh et al., 1999) and inhibits long-term potentiation (Katsuki et al., 1990; Bellinger et al., 1993; Cunningham et al., 1996; O'Connor and Coogan, 1999; Vereker et al., 2000) though the mechanisms are largely unknown. Maternal infection is proposed to cause cytokine imbalance in fetal brain as well as maternal serum and alter fetal development and subsequent behavior and/or cognitive function in the offspring (Deverman and Patterson, 2009; Patterson, 2009). We here showed that IL-1RAcP and its neuronal isoform IL-1RAcPb act as trans-synaptic cell adhesion molecules and mediate synapse formation. The mode of action of IL-1RAcP in synapse formation is distinct from the well known signaling role of IL-1RAcP in inflammation (Dinarello, 2009; Sims and Smith, 2010) (Fig. 11). Our results reveal an interesting molecular link between immune systems and synapse formation in the brain.
The key finding of the present investigation is the identification of the essential subunit of receptors for IL-1 family cytokines as a synaptogenic organizer in the brain. Knockdown of IL$1 \mathrm{RAcP}$ isoforms in cortical neurons suppressed synapse formation as indicated by the reduction of staining signals for presynaptic Bassoon and by the decrease of number of dendritic protrusions. Both phenotypes were rescued by the neuronal isoform IL-1RAcPb, while only presynaptic Bassoon signals were recovered by IL-1RAcP. Thus, both IL-1RAcP and IL-1RAcPb have the ability to induce presynaptic differentiation, while the spinogenic activity is specific for the neuronal isoform. The ECD of IL-1RAcPb that is identical to that of IL-1RAcP was sufficient to induce presynaptic differentiation. We identified PTP $\delta$ as a ligand for IL-1RAcP isoforms, while LAR and PTP $\sigma$, other members of 2A-type RPTP proteins, showed weak binding activities. The presynaptic differentiation induced by IL-1RAcP isoforms was severely suppressed in the cultured cortical neurons from $\mathrm{PTP} \delta$ knock-out mice. These results suggest that IL-1RAcP isoforms induce presynaptic differentiation by interacting with presynaptic PTP $\delta$.

Although IL-1RAcP is the essential component of the signaling-competent receptor complexes for IL-1 family cytokines, IL-1RAcP by itself does not interact with the cytokines (Greenfeder et al., 1995; Palmer et al., 2008). Thus, to our knowledge, the present investigation is the first report of the identifica- 
tion of IL-1RAcP ligand. We showed the cell adherent interaction between IL$1 \mathrm{RAcP}$ and PTP $\delta$ required neither IL-1 family cytokines nor the ligand-recognition receptors for the IL-1 family cytokines. Thus, our results revealed the role of IL-1RAcP as a synaptic cell adhesion molecule. Our finding raises an intriguing possibility that the cell adherent interaction between IL-1RAcP and PTP $\delta$ may also play a role by modulating signaling of IL-1 family cytokines or mediating signaling independent of them in various tissues where both molecules are expressed (Mizuno et al., 1994; Greenfeder et al., 1995). It will be interesting to note that the PTPRD gene is often inactivated in cancers including melanoma, head and neck cancer, and lung cancer (Julien et al., 2011) while upregulation of IL-1 signaling is implicated in the progression and metastasis of these cancers (Dinarello, 2010).

We also showed that the PTP $\delta$ A3-ECDinduced postsynaptic differentiation was suppressed in cultured neurons from IL1RAcP knock-out mice and the expression of IL-1RAcPb but not IL-1RAcP recovered the postsynaptic differentiation. These results suggest that IL-1RAcPb bidirectionally regulates synapse formation of cortical neurons, while IL-1RAcP induces unidirectionally presynaptic differentiation. Since magnetic beads coated with IL-1RAcP-ECD were sufficient to induce presynaptic differentiation in cortical neurons, IL-1RAcP isoforms appear to require no other receptor components for the IL-1 family cytokines to exert their synaptogenic activities. On the other hand, the ECD of IL-1R1, IL-1R2, ST2 or IL-1Rrp2 coated on magnetic beads failed to show any synaptogenic activity in cultured cortical neurons. Both IL-1RAcP isoforms showed the ability to induce presynaptic differentiation, whereas the abilities to increase the number of dendritic protrusions and to induce Shank2 accumulation were specific for IL-1RAcPb. Because IL-1RAcPb differs from IL-1RAcP only in the C-terminal region ( $\mathrm{Lu}$ et al., 2008; Smith et al., 2009), it is likely that the C-terminal 240 aa sequence specific for IL-1RAcPb is responsible for the spinogenic activity and the activity to induce postsynaptic differentiation. Among ligand-binding subunits for IL-1 family cytokines, IL-1R1 is a major subunit expressed in neural cells (Yabuuchi et al., 1994; Ericsson et al., 1995; Andre et al., 2005). The association of IL-1R1 with IL-1RAcPb requires IL-1 $\beta$ (Smith et al., 2009). However, PTP $\delta A 3$-coated beads induced postsynaptic differentiation through IL-1RAcPb without the addition of any interleukins. Thus, it seems unlikely that IL-1RAcPb mediates spinogenesis and postsynaptic differentiation in association with IL-1R1, although a possible interaction of IL-1RAcPb with an unknown partner is not completely excluded.

IL1RAPL1 is classified as a member of the IL-1 receptor family, but has no activity to mediate immune signals as a component

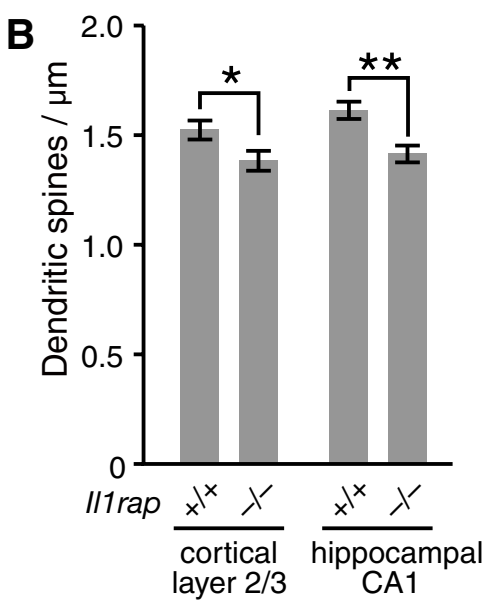

Figure 10. Decreased spine densities of cortical layer 2/3 and hippocampal CA1 pyramidal neurons in IL-1RAcP knock-out mice. $\boldsymbol{A}$, Dil labeling of basal dendrites of cortical layer $2 / 3$ and hippocampal CA1 pyramidal neurons in wild-type $\left(/ / 1 \mathrm{rap}^{+/+}\right)$and ( ) mice. $B$, Spine densities of basal dendrites of cortical layer 2/3 and hippocampal CA1 pyramidal ectively from 2 animals). All values represent mean \pm SEM, ${ }^{*} p<0.05$ and ${ }^{* *} p<0.01$, respectively; Student's t test. Scale bar,

\section{Immune regulation and inflammation}

\section{Synapse formation}

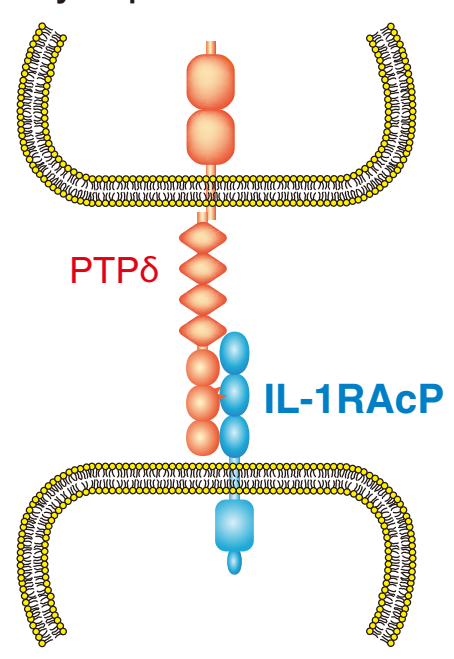

Figure 11. A proposed model for the role of IL-1RAcP as a neuronal cell adhesion molecule. IL-1RAcP acts as a trans-synaptic cell adhesion molecule and mediates neuronal synaptogenesis (right). The mode of action of IL-1RAcP in synaptogenesis is distinct from the well known signaling role of IL-1RAcP in immune regulation and inflammation (left).

of the receptors for IL-1 family cytokines (Born et al., 2000; Sims and Smith, 2010). We recently showed that IL1RAPL1 organizes synapse formation of mouse cortical neurons through transsynaptic interaction with PTP $\delta$ (Yoshida et al., 2011). Since IL1RAPL1 is responsible for nonsyndromic MR and is associated with autism (Carrié et al., 1999; Piton et al., 2008), we propose that the impairment of synapse formation may underlie the pathogenesis of certain forms of MR and autism. In addition, IL1RAPL2, a close relative of IL1RAPL1 sharing 61.5\% amino acid sequence identity, showed a weak synaptogenic activity in fibroblast-neuron mixed culture assay (data not shown). In contrast, IL-1RAcP showed a stronger synaptogenic activity than IL1RAPL1 (data not shown), while IL-18RAcP showed no detectable activity. Interestingly with this respect, IL-1RAcP shares higher amino acid sequence identities in the ECD with IL1RAPL1 and IL1RAPL2 (30.3\% and 32.5\%, respectively) than IL-18RAcP 
(17.1\%) and the ligand-binding subunits of the IL-1R family (16.4-25.5\%). Amino-acid sequence identity between IL-1RAcP and IL1RAPL1/2 is much higher in the first Ig-like domain (50.0$52.2 \%)$ than in the second or third Ig-like domain (22.2-35.8\%). Thus, the first Ig-like domain of these proteins may be important for their synaptogenic activity.

The developing mouse brain expressed at least 6 variants of $\mathrm{PTP} \delta$ derived from alternative splicing at 3 small exons encoding meA and meB peptides. The meB peptide of PTP $\delta$ is essential for binding to IL-1RAcP. On the other hand, the meA peptide of PTP $\delta$ was critical for the interaction with IL1RAPL1 (Yoshida et al., 2011). Thus, the interactions with PTP $\delta$ variants are differential between IL-1RAcP and IL1RAPL1. The mini-exon peptides of PTP $\delta$ represent protein codes for the specificity of synapse formation through trans-synaptic interaction with IL-1RAcP and IL1RAPL1.

LAR, PTP $\delta$ and PTP $\sigma$ share similar domain structures and constitute 2A-type RPTPs (Tonks, 2006). IL-1RAcP isoforms interacted with $\mathrm{PTP} \sigma$ and LAR in addition to PTP $\delta$. In contrast, IL1RAPL1 interacted selectively with specific splice variants of PTP $\delta$ to induce excitatory synapse formation (Yoshida et al., 2011). Interestingly, Takahashi et al. (2011) reported that TrkC selectively interacts with PTP $\sigma$ among 2A-type RPTPs and induces excitatory synapse formation bidirectionally. On the other hand, NGL-3 interacts with all members of 2A-type RPTPs and induces presynaptic differentiation of cultured rat hippocampal neurons (Woo et al., 2009; Kwon et al., 2010). Furthermore, PTP $\sigma$ regulates the synapse number of zebrafish olfactory sensory neurons (Chen et al., 2011). The selective formation of the huge numbers of synapses in the brain would require specific synaptogenic codes. Presynaptic 2A-type RPTPs interact with diverse postsynaptic cell adhesion molecules in a subtype- and variant-specific manner. Recent studies also revealed that presynaptic NRXNs interact with postsynaptic NLGNs, LRRTM2 and Cbln1-GluR $\delta 2$, in a variant-specific manner (Südhof, 2008; de Wit et al., 2009; Ko et al., 2009; Uemura et al., 2010). Thus, 2A-type RPTPs and NRXNs may provide presynaptic molecular codes for synapse formation.

\section{References}

Andre R, Lerouet D, Kimber I, Pinteaux E, Rothwell NJ (2005) Regulation of expression of the novel IL-1 receptor family members in the mouse brain. J Neurochem 95:324-330.

Aubert A, Vega C, Dantzer R, Goodall G (1995) Pyrogens specifically disrupt the acquisition of a task involving cognitive processing in the rat. Brain Behav Immun 9:129-148.

Avital A, Goshen I, Kamsler A, Segal M, Iverfeldt K, Richter-Levin G, Yirmiya R (2003) Impaired interleukin-1 signaling is associated with deficits in hippocampal memory processes and neural plasticity. Hippocampus 13:826-834.

Bellinger FP, Madamba S, Siggins GR (1993) Interleukin- $\beta$ inhibits synaptic strength and long-term potentiation in the rat CA1 hippocampus. Brain Res 628:227-234.

Born TL, Smith DE, Garka KE, Renshaw BR, Bertles JS, Sims JE (2000) Identification and characterization of two members of a novel class of the interleukin-1 receptor (IL-1R) family. Delineation of a new class of IL1R-related proteins based on signaling. J Biol Chem 275:29946-29954.

Carrié A, Jun L, Bienvenu T, Vinet MC, McDonell N, Couvert P, Zemni R, Cardona A, Van Buggenhout G, Frints S, Hamel B, Moraine C, Ropers HH, Strom T, Howell GR, Whittaker A, Ross MT, Kahn A, Fryns JP, Beldjord C, et al. (1999) A new member of the IL-1 receptor family highly expressed in hippocampus and involved in X-linked mental retardation. Nat Genet 23:25-31.

Chen X, Yoshida T, Sagara H, Mikami Y, Mishina M 2011 Protein tyrosine phosphatase $\sigma$ regulates the synapse number of zebrafish olfactory sensory neurons. J Neurochem 119:532-543.
Cullinan EB, Kwee L, Nunes P, Shuster DJ, Ju G, McIntyre KW, Chizzonite RA, Labow MA (1998) IL-1 receptor accessory protein is an essential component of the IL-1 receptor. J Immunol 161:5614-5620.

Cunningham AJ, Murray CA, O’Neill LAJ, Lynch MA, O’Connor JJ (1996) Interleukin- $1 \beta$ (IL- $1 \beta$ ) and tumour necrosis factor (TNF) inhibit longterm potentiation in the rat dentate gyrus in vitro. Neurosci Lett 203: $17-20$.

Dascombe MJ (1985) The pharmacology of fever. Prog Neurobiol 25: 327-373.

Deverman BE, Patterson PH (2009) Cytokines and CNS development. Neuron 64:61-78.

de Wit J, Sylwestrak E, O'Sullivan ML, Otto S, Tiglio K, Savas JN, Yates JR 3rd, Comoletti D, Taylor P, Ghosh A (2009) LRRTM2 interacts with Neurexin1 and regulates excitatory synapse formation. Neuron 64:799-806.

Dinarello CA (2009) Immunological and inflammatory functions of the interleukin-1 family. Annu Rev Immunol 27:519-550.

Dinarello CA (2010) Why not treat human cancer with interleukin-1 blockade? Cancer Metastasis Rev 29:317-329.

Ericsson A, Liu C, Hart RP, Sawchenko PE (1995) Type 1 interleukin-1 receptor in the rat brain: distribution, regulation, and relationship to sites of IL-1-induced cellular activation. J Comp Neurol 361:681-698.

Friedman WJ (2001) Cytokines regulate expression of the type 1 interleukin-1 receptor in rat hippocampal neurons and glia. Exp Neurol 168:23-31.

Gibertini M, Newton C, Friedman H, Klein TW (1995) Spatial learning impairment in mice infected with Legionella pneumophila or administered exogenous interleukin-1 $\beta$. Brain Behav Immun 9:113-128.

Goshen I, Yirmiya R (2009) Interleukin-1 (IL-1): a central regulator of stress responses. Front Neuroendocrinol 30:30-45.

Greenfeder SA, Nunes P, Kwee L, Labow M, Chizzonite RA, Ju G (1995) Molecular cloning and characterization of a second subunit of the interleukin 1 receptor complex. J Biol Chem 270:13757-13765.

Horai R, Asano M, Sudo K, Kanuka H, Suzuki M, Nishihara M, Takahashi M, Iwakura Y (1998) Production of mice deficient in genes for interleukin (IL)- $1 \alpha$, IL- $1 \beta$, IL- $1 \alpha / \beta$, and IL- 1 receptor antagonist shows that IL- $1 \beta$ is crucial in turpentine-induced fever development and glucocorticoid secretion. J Exp Med 187:1463-1475.

Julien SG, Dubé N, Hardy S, Tremblay ML (2011) Inside the human cancer tyrosine phosphatome. Nat Rev Cancer 11:35-49.

Katsuki H, Nakai S, Hirai Y, Akaji K, Kiso Y, Satoh M (1990) Interleukin-1 $\beta$ inhibits long-term potentiation in the CA3 region of mouse hippocampal slices. Eur J Pharmacol 181:323-326.

Kim BG, Dai HN, McAtee M, Vicini S, Bregman BS (2006) Remodeling of synaptic structures in the motor cortex following spinal cord injury. Exp Neurol 198:401-415.

Ko J, Fuccillo MV, Malenka RC, Südhof TC (2009) LRRTM2 functions as a neurexin ligand in promoting excitatory synapse formation. Neuron 64:791-798.

Krueger JM (2008) The role of cytokines in sleep regulation. Curr Pharm Des 14:3408-3416.

Kwon SK, Woo J, Kim SY, Kim H, Kim E (2010) Trans-synaptic adhesions between netrin-G ligand-3 (NGL-3) and receptor tyrosine phosphatases LAR, protein-tyrosine phosphatase $\delta$ (PTP $\delta$ ), and PTP $\sigma$ via specific domains regulate excitatory synapse formation. J Biol Chem 285:13966-13978.

Lu HL, Yang CY, Chen HC, Hung CS, Chiang YC, Ting LP (2008) A novel alternatively spliced interleukin-1 receptor accessory protein mIL1RAcP687. Mol Immunol 45:1374-1384.

Matsuki T, Horai R, Sudo K, Iwakura Y (2003) IL-1 plays an important role in lipid metabolism by regulating insulin levels under physiological conditions. J Exp Med 198:877-888.

Miura E, Fukaya M, Sato T, Sugihara K, Asano M, Yoshioka K, Watanabe M (2006) Expression and distribution of JNK/SAPK-associated scaffold protein JSAP1 in developing and adult mouse brain. J Neurochem 97:1431-1446.

Miyazaki T, Fukaya M, Shimizu H, Watanabe M (2003) Subtype switching of vesicular glutamate transporters at parallel fibre-Purkinje cell synapses in developing mouse cerebellum. Eur J Neurosci 17:2563-2572.

Mizuno K, Hasegawa K, Ogimoto M, Katagiri T, Yakura H (1994) Developmental regulation of gene expression for the MPTP $\delta$ isoforms in the central nervous system and the immune system. FEBS Lett 355:223-228.

O'Connor JJ, Coogan AN (1999) Actions of the pro-inflammatory cytokine IL-1 $\beta$ on central synaptic transmission. Exp Physiol 84:601-614. 
Oitzl MS, van Oers H, Schöbitz B, de Kloet ER (1993) Interleukin-1 $\beta$, but not interleukin-6, impairs spatial navigation learning. Brain Res 613: $160-163$.

O'Neill LA (2008) The interleukin-1 receptor/Toll-like receptor superfamily: 10 years of progress. Immunol Rev 226:10-18.

Palmer G, Lipsky BP, Smithgall MD, Meininger D, Siu S, Talabot-Ayer D, Gabay C, Smith DE (2008) The IL-1 receptor accessory protein (AcP) is required for IL-33 signaling and soluble AcP enhances the ability of soluble ST2 to inhibit IL-33. Cytokine 42:358-364.

Patterson PH (2009) Immune involvement in schizophrenia and autism: etiology, pathology and animal models. Behav Brain Res 204:313-321.

Piton A, Michaud JL, Peng H, Aradhya S, Gauthier J, Mottron L, Champagne N, Lafrenière RG, Hamdan FF; S2D team, Joober R, Fombonne E, Marineau C, Cossette P, Dubé MP, Haghighi P, Drapeau P, Barker PA, Carbonetto S, Rouleau GA (2008) Mutations in the calcium-related gene IL1RAPL1 are associated with autism. Hum Mol Genet 17:3965-3974.

Pugh CR, Nguyen KT, Gonyea JL, Fleshner M, Wakins LR, Maier SF, Rudy JW (1999) Role of interleukin-1 beta in impairment of contextual fear conditioning caused by social isolation. Behav Brain Res 106:109-118.

Pulido R, Serra-Pagès C, Tang M, Streuli M (1995) The LAR/PTP $\delta / P T P \sigma$ subfamily of transmembrane protein-tyrosine-phosphatases: multiple human LAR, PTP $\delta$, and PTP $\sigma$ isoforms are expressed in a tissue-specific manner and associate with the LAR-interacting protein LIP.1. Proc Natl Acad Sci U S A 92:11686-11690.

Rothwell NJ, Luheshi GN (2000) Interleukin 1 in the brain: biology, pathology and therapeutic target. Trends Neurosci 23:618-625.

Scheiffele P, Fan J, Choih J, Fetter R, Serafini T (2000) Neuroligin expressed in nonneuronal cells triggers presynaptic development in contacting axons. Cell 101:657-669.

Sims JE, Smith DE (2010) The IL-1 family: regulators of immunity. Nat Rev Immunol 10:89-102.

Smith DE, Lipsky BP, Russell C, Ketchem RR, Kirchner J, Hensley K, Huang Y, Friedman WJ, Boissonneault V, Plante MM, Rivest S, Sims JE (2009) A central nervous system-restricted isoform of the interleukin-1 receptor accessory protein modulates neuronal responses to interleukin-1. Immunity 30:817-831.

Südhof TC (2008) Neuroligins and neurexins link synaptic function to cognitive disease. Nature 455:903-911.

Takahashi H, Sekino Y, Tanaka S, Mizui T, Kishi S, Shirao T (2003) Drebrin-dependent actin clustering in dendritic filopodia governs synap- tic targeting of postsynaptic density-95 and dendritic spine morphogenesis. J Neurosci 23:6586-6595.

Takahashi H, Arstikaitis P, Prasad T, Bartlett TE, Wang YT, Murphy TH, Craig AM (2011) Postsynaptic TrkC and presynaptic PTP $\sigma$ function as a bidirectional excitatory synaptic organizing complex. Neuron 69: 287-303.

Tonks, NK (2006) Protein tyrosine phosphatases: from genes, to function, to disease. Nat Rev Mol Cell Biol 7:833-846.

Uemura T, Mishina M (2008) The amino-terminal domain of glutamate receptor $\delta 2$ triggers presynaptic differentiation. Biochem Biophys Res Commun 377:1315-1319.

Uemura T, Mori H, Mishina M (2004) Direct interaction of GluR $\delta 2$ with Shank scaffold proteins in cerebellar Purkinje cells. Mol Cell Neurosci 26:330-341.

Uemura T, Lee SJ, Yasumura M, Takeuchi T, Yoshida T, Ra M, Taguchi R, Sakimura K, Mishina M (2010) Trans-synaptic interaction of GluR $\delta 2$ and neurexin through Cbln1 mediates synapse formation in the cerebellum. Cell 141:1068-1079.

Uetani N, Kato K, Ogura H, Mizuno K, Kawano K, Mikoshiba K, Yakura H, Asano M, Iwakura Y (2000) Impaired learning with enhanced hippocampal long-term potentiation in PTP $\delta$-deficient mice. EMBO J 19:2775-2785.

Vereker E, O'Donnell E, Lynch MA (2000) The inhibitory effect of interleukin- $1 \beta$ on long-term potentiation is coupled with increased activity of stress-activated protein kinases. J Neurosci 20:6811-6819.

Woo J, Kwon SK, Choi S, Kim S, Lee JR, Dunah AW, Sheng M, Kim E (2009) Trans-synaptic adhesion between NGL-3 and LAR regulates the formation of excitatory synapses. Nat Neurosci 12:428-437.

Yabuuchi K, Minami M, Katsumata S, Satoh M (1994) Localization of type I interleukin-1 receptor mRNA in the rat brain. Brain Res Mol Brain Res 27:27-36.

Yoshida T, Mishina M (2008) Zebrafish orthologue of mental retardation protein IL1RAPL1 regulates presynaptic differentiation. Mol Cell Neurosci 39:218-228.

Yoshida T, Yasumura M, Uemura T, Lee SJ, Ra M, Taguchi R, Iwakura Y, Mishina M (2011) IL-1 receptor accessory protein-like 1 associated with mental retardation and autism mediates synapse formation by transsynaptic interaction with protein tyrosine phosphatase $\delta$. J Neurosci 31: 13485-13499. 